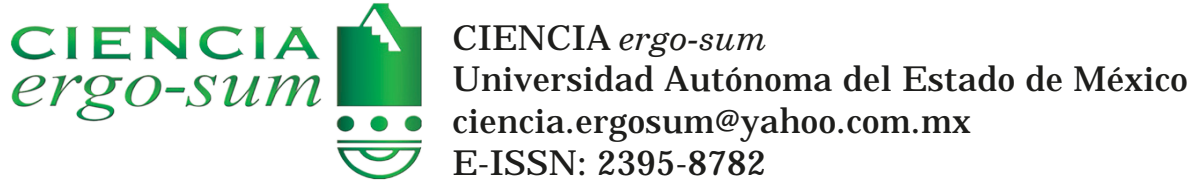

\title{
Evaluación de la vulnerabilidad ante eventos climáticos extremos en La Paz, Baja California Sur, México
}

Turrén-Cruz, Thalía; Benegas, Laura; Gutiérrez-Montes, Isabel y Brenes, Christian

Evaluación de la vulnerabilidad ante eventos climáticos extremos en La Paz, Baja California Sur, México

CIENCIA ergo-sum, vol. 26, núm. 1, marzo-junio 2019|e41

Universidad Autónoma del Estado de México, México

Esta obra está bajo una Licencia Creative Commons Atribución-N oComercial-SinDerivar 4.0 Internacional.

Turrén-Cruz, T., Benegas, L., Gutiérrez-Montes, I. y Brenes, C. (2018). Agroquímicos organofosforados y su potencial daño en la salud de trabajadores agrícolas del campo sonorense. CIE NCIA ergo-sum, 26(1). https://doi.org/10.30878/ ces.v26n1a6 


\title{
Evaluación de la vulnerabilidad ante eventos climáticos extremos en La Paz, Baja California Sur, México
}

Evaluation of vulnerability to extreme climate events in La Paz, Baja California Sur; México

Thalia Turrén-Cruz

Centro Agronómico Tropical de Investigación y Enseñanza (CATIE), Costa Rica

Recepción: 14 de febrero de 2017

thalia.turren@catie.ac.cr

Aprobación: 24 de octubre de 2017

\author{
Laura Benegas \\ Centro Agronómico Tropical de Investigación y Enseñanza(CATIE), Costa Rica \\ ibenegas@catie.ac.cr \\ Isabel Gutiérrez-Montes \\ Centro Agronómico Tropical de Investigación y Enseñanza (CATIE), Costa Rica \\ igutierrez@catie.ac.cr \\ Christian Brenes \\ Centro Agronómico Tropical de Investigación y Enseñanza (CATIE), Costa Rica \\ cbrenes@catie.ac.cr
}

\section{RESUMEN}

Se evalúa la vulnerabilidad ante la escasez de agua y sequía en La Paz, Baja California Sur. Se utilizó el Marco de Capitales de la Comunidad (MCC) para estimar la vulnerabilidad como un sistema integrado por siete subsistemas: humano, social, político, financiero, físico, natural y cultural mediante un análisis de situación biofísica, cálculo de índices, así como la identificación de medidas y estrategias que ayuden a mejorar la capacidad adaptativa de la comunidad. Se obtiene la vulnerabilidad integral que se representó espacialmente en la zona de estudio. La Paz presenta una vulnerabilidad media y es necesario fortalecer las capacidades de la comunidad priorizando el trabajo comunidad-gobierno no sólo para hacer frente a este tipo de eventos sino para mejorar también la calidad de vida. Palabras Clave: sequía, marco de capitales de la comunidad, vulnerabilidad, índices.

\section{Abstract}

A vulnerability assessment due to water scarcity and drought in the city of $\mathrm{La} \mathrm{Paz}(\mathrm{BCS})$ is presented. The community capitals framework (CFF) is used to estimate vulnerability as a system integrated by 7 sub-systems: human, social, political, financial, physical, natural and cultural, through a biophysical situation analysis, index calculation, and the identification of strategies that help to improve the adaptive capacity of the community. The vulnerability obtained, is spatially represented in the city of La Paz, it represents a medium vulnerability, and it is necessary to strengthen community capacities prioritizing community-government work, not just to face these events but also to improve community's quality of life.

KEYWORDS: drought, community capitals framework, vulnerability, index.

\section{INTRODUCCIÓN}

La Paz Baja, California Sur, es una ciudad ubicada en una de las regiones más áridas y secas de México. Esta característica ha desencadenado problemas de disponibilidad de agua, sobre todo la superficial, que con el paso de los años se han agravado (Fonseca y Ramírez, 2014).

El agua subterránea es la principal fuente de abastecimiento de la ciudad y su extracción aumenta rápidamente debido al incremento de los pobladores. Por otro lado, la escasa precipitación determinada por su ubicación geográfica resulta en un limitado aprovechamiento del agua superficial (Escolero y Torres-Onofre, 2007). 
Durante la época de ciclones se recibe una cantidad importante de precipitación; sin embargo, los volúmenes de lluvia no son aprovechados para resolver los problemas de disponibilidad de agua que presenta la región debido a la falta de infraestructura y, como consecuencia, los eventos extraordinarios de lluvia y escurrimiento causan daños severos (Fonseca y Ramírez, 2014; Salas y Jiménez, 2013).

Además de la problemática de la disponibilidad, se ha reportado que el agua del acuífero La Paz posee ciertos niveles de salinidad sobre todo la más cercana a la superficie, de 10 a $15 \mathrm{~m}$ de profundidad, impactando en su calidad, por lo que agudiza aún más la disponibilidad del recurso. Este aspecto es analizado en estudios de Montalvo (2010), SAPA (2010) y (2013), CNA (2002) y CONAGUA y UNAM (2010), los cuales recopilan Fonseca y Ramírez (2014) .

Los niveles de agua contenidas en el acuífero presentan un desbalance provocado porque se extrae más de la que se recarga. Las cifras dadas por la Comisión Nacional del Agua (CONAGUA) evidencian lo anterior, ya que se evapora más agua de la que llueve, tiende a sobreexplotarse el recurso, hay contaminación y existe poca ocurrencia de flujos superficiales en la zona. Actualmente, el gobierno municipal ha gestionado algunas iniciativas en las cuales se encuentran tres convenios realizados con la CONAGUA: a) la construcción de infraestructura necesaria, como una planta de tratamiento de aguas residuales, $b$ ) un acueducto y $c$ ) una planta desalinizadora (SEGOB, 2013).

Para dar respuesta a la problemática del recurso hídrico en La Paz es importante considerar su vulnerabilidad ante los efectos del cambio climático como la escasez y la sequía. La vulnerabilidad es definida como "el grado por el cual un sistema es susceptible o incapaz de enfrentarse a efectos adversos del cambio climático, incluidas la variabilidad y los extremos del clima" (IPCC, 2001). Incluso, se hace necesaria la gestión integral de los recursos hídricos, un proceso que promueve la gestión y desarrollo del agua, suelo y otros recursos con el fin de maximizar los resultados económicos y el bienestar social de forma equitativa sin comprometer la sostenibilidad de los ecosistemas vitales (ONU, 2014).

A fin de evaluar la vulnerabilidad de forma integral, se usa como enfoque metodológico y conceptual el Marco de Capitales de la Comunidad (MCC) diseñado por Flora et al. (2016) y aplicado por Gutiérrez et al. (2014), el cual plantea que cada comunidad, no importa lo marginada o pobre que sea, cuenta con recursos que puede disponer para promover su desarrollo. Estos recursos se categorizan en tres: $a$ ) los que pueden ser consumidos, $b$ ) los que pueden ser almacenados y c) los que pueden invertirse para crear más recursos; estos últimos se conceptualizan como capitales, que a su vez se dividen en dos grandes grupos: a) capitales humanos y b) capitales materiales.

Con estos elementos, el objetivo de este artículo es estimar la vulnerabilidad ante la escasez de agua y sequía en la ciudad de La Paz mediante el análisis de su situación biofísica histórico-actual, la estimación de la vulnerabilidad y la identificación de medidas y estrategias que ayuden a mejorar la capacidad adaptativa de la comunidad ante los efectos del cambio climático. Este análisis se basó en el Marco de Capitales de la Comunidad (MCC), por lo cual el propósito es evidenciar que existe una vulnerabilidad integral diferenciada según la ubicación de las diferentes zonas de La Paz y que puede ser explicada por los capitales de la comunidad.

\section{Materiales y Métodos}

\section{1. Zona de estudio}

La Paz se encuentra en el estado de Baja California Sur (mapa 1). Está ubicada entre las coordenadas $23^{\circ} 6^{\prime} 45^{\prime \prime}$ y $25^{\circ} 5^{\prime}$ 2" latitud norte y los $111^{\circ} 41^{\prime} 52^{\prime \prime}$ y $109^{\circ} 41^{\prime} 44^{\prime \prime}$ longitud oeste. Cuenta con una extensión territorial de 20 $274 \mathrm{~km}^{2}$ (20.76\% del territorio estatal) y una población de 251178 habitantes, de la cual 50.2\% corresponde a hombres y $49.8 \%$ a mujeres; además, de 100\% de la población, de acuerdo con el INEGI (2011), 86\% es urbana y $14 \%$ restante es rural. Entre las principales actividades económicas se encuentran el turismo, el comercio y agricultura. 


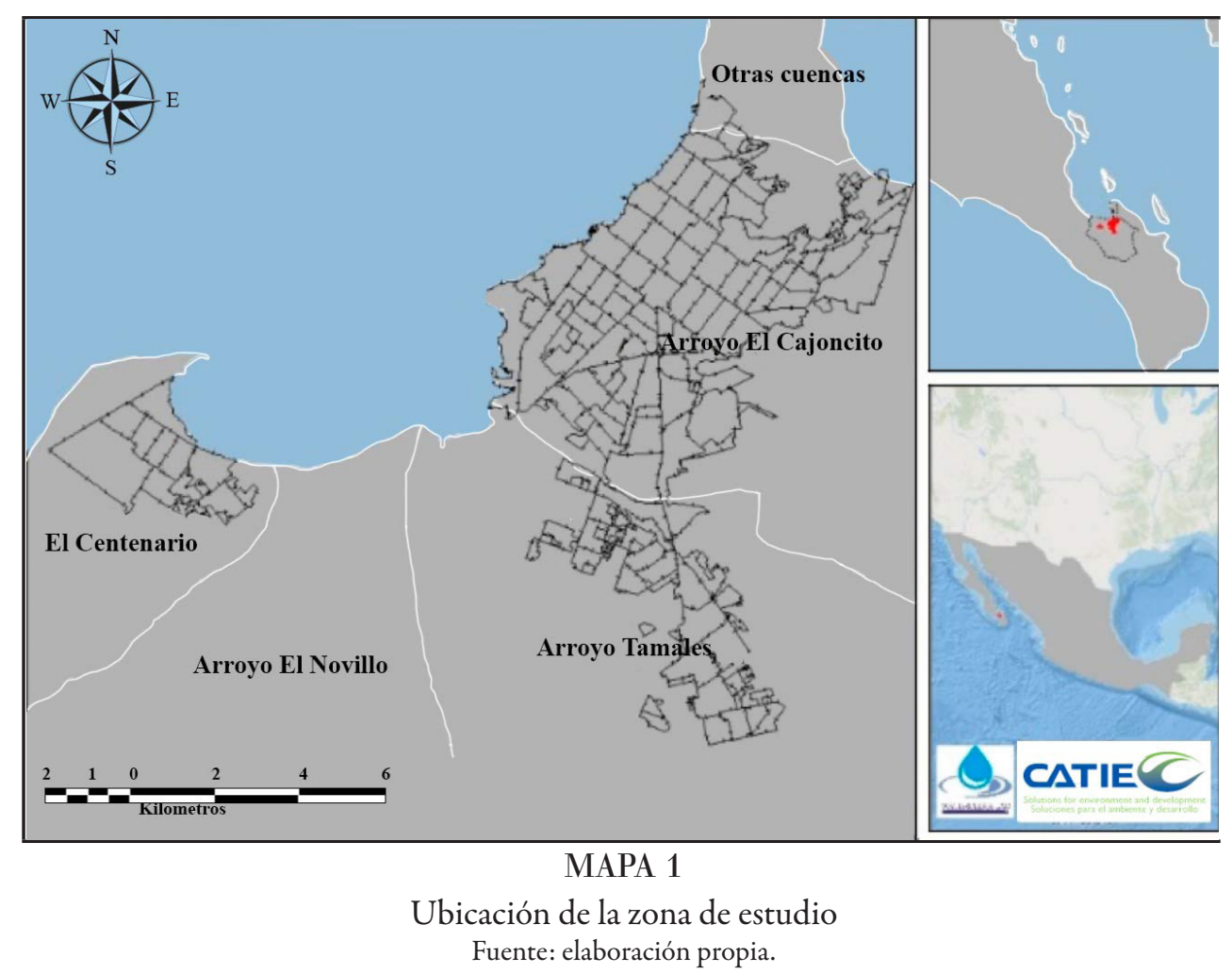

De acuerdo con información del INEGI (2011), predomina el clima muy seco (92\%), pero también hay presencia de clima seco y semiseco (7\%) y templado subhúmedo (1\%). La temperatura promedio más alta (35 ${ }^{\circ} \mathrm{C}$ ) se presenta en los meses de julio y agosto, la temperatura media anual es 18 a $22{ }^{\circ} \mathrm{C}$ y la más baja es de $9{ }^{\circ} \mathrm{C}$, que se registra en enero. Las lluvias son muy escasas y se presentan durante el verano. La precipitación total anual promedio en el estado es menor a $200 \mathrm{~mm}$; debido a esto, la poca actividad agrícola que se practica es de riego. Se cultiva algodón, trigo, alfalfa verde, frijol, jitomate, cártamo, chile verde y papa (INEGI, 2011)

\subsection{Definición de indicadores}

La selección de indicadores para evaluar la vulnerabilidad se basó en las metodologías de Vulnerabilidad y adaptación al cambio climático de los pobladores rurales de la planicie costera central de El Salvador de Aguilar (2007), Vulnerabilidad global de Wilches-Chaux (1993), el enfoque de vulnerabilidad integral analizado por Lampis (2013) y el Marco de Capitales de la Comunidad (MCC) aplicado en México por Gutierréz-Montes et al. (2014). Se definieron 42 indicadores en total (tabla 1), divididos en siete capitales: humano, social, político, financiero, físico, natural y cultural que ayudan a definir el diseño de la encuesta para la recolección de datos en campo.

Los indicadores se validaron mediante una encuesta en línea aplicada a diez actores clave de la zona con la herramienta SurveyMonkey. A cada participante se le preguntó cuáles consideraba necesarios para evaluar la vulnerabilidad de La Paz. Estos se clasificaron de acuerdo con la necesidad: baja (1), media (2) y alta (3). Además, se les cuestionó si lo consideraban innecesario para quitarlo de la lista y también se brindó la opción de sugerir algún otro que sí lo fuera; de esta forma, se obtuvieron las ponderaciones de importancia para los indicadores.

\subsection{Protocolo para las encuestas}

El protocolo consistió en presentar la investigación, el consentimiento informado, una serie de 60 preguntas y los datos de contacto del investigador (Sibelet et al., 2013). Las preguntas de la encuesta siguen un orden lógico con 
la intención de dar respuesta a cada uno de los indicadores asociados a los capitales de la comunidad necesarios para la investigación (tabla 2). Hay por lo menos una pregunta que ayuda a obtener información sobre cada indicador de interés.

TABLA 1

Indicadores por capital

\begin{tabular}{|c|c|c|c|}
\hline Capital & Indicador & Capital & Indicador \\
\hline \multirow{9}{*}{$\begin{array}{l}\text { Humano } \\
\text { (IVH) }\end{array}$} & Población con servicio de agua & \multirow{6}{*}{$\begin{array}{l}\text { Financiero } \\
\text { (IVFN) }\end{array}$} & Acceso a programas de gobierno \\
\hline & $\begin{array}{l}\text { Densidad poblacional (número de } \\
\text { habitantes por casa) }\end{array}$ & & Acceso a remesas \\
\hline & $\begin{array}{l}\text { Enfermedades asociadas al consumo de } \\
\text { agua }\end{array}$ & & Acceso a crédito \\
\hline & Enfermedades asociadas a la sequía & & Cambio de ingresos por evento climático \\
\hline & $\begin{array}{l}\text { Enfermedades asociadas a la escasez de } \\
\text { agua }\end{array}$ & & Costo del servicio \\
\hline & $\begin{array}{l}\text { Conocimiento sobre acciones frente a un } \\
\text { evento climático }\end{array}$ & & Costo por falta de agua \\
\hline & Migración & \multirow{4}{*}{$\begin{array}{l}\text { Físico } \\
\text { (IVFS) }\end{array}$} & Disponibilidad del servicio \\
\hline & Inmigración & & $\begin{array}{l}\text { Infraestructura usada en caso de evento } \\
\text { climático (albergues) }\end{array}$ \\
\hline & $\begin{array}{l}\text { Asistencia a capacitación sobre eventos } \\
\text { climáticos }\end{array}$ & & $\begin{array}{l}\text { Infraestructura afectada en caso de evento } \\
\text { climático }\end{array}$ \\
\hline \multirow{5}{*}{$\begin{array}{l}\text { Social } \\
\text { (IVS) }\end{array}$} & $\begin{array}{l}\text { Pertenencia a organizaciones de la } \\
\text { comunidad }\end{array}$ & & Fuentes de abastecimiento \\
\hline & $\begin{array}{l}\text { Existencia de organizaciones en la } \\
\text { comunidad }\end{array}$ & \multirow{7}{*}{$\begin{array}{l}\text { Natural } \\
\text { (IVN) }\end{array}$} & Percepción de la vulnerabilidad del suelo \\
\hline & $\begin{array}{l}\text { Percepción sobre el nivel de organización } \\
\text { en la comunidad }\end{array}$ & & Percepción de la vulnerabilidad del agua \\
\hline & $\begin{array}{l}\text { Existencia de redes de apoyo en la } \\
\text { comunidad }\end{array}$ & & $\begin{array}{l}\text { Percepción de la vulnerabilidad de la } \\
\text { vegetación }\end{array}$ \\
\hline & Participación en la formulación de planes & & $\begin{array}{l}\text { Percepción de la vulnerabilidad de los } \\
\text { animales }\end{array}$ \\
\hline \multirow{8}{*}{$\begin{array}{l}\text { Político } \\
\text { (IVP) }\end{array}$} & Gestión de las autoridades locales & & Percepción de cambios en el clima \\
\hline & Respuesta del gobierno (local) & & Contaminación del agua \\
\hline & Presencia de instituciones a nivel local & & Recurrencia de eventos climáticos \\
\hline & $\begin{array}{l}\text { Capacidad de reacción de protección civil u } \\
\text { otro tipo de apoyo }\end{array}$ & \multirow{5}{*}{$\begin{array}{l}\text { Cultural } \\
\text { (IVC) }\end{array}$} & Comunicación ante un evento climático \\
\hline & Regulación del agua & & Usos del agua \\
\hline & Comité de cuencas & & Reúso del agua \\
\hline & & & Recurrencia a autoridades por falta de agua \\
\hline & & & Cambios en la forma de vida \\
\hline
\end{tabular}

Fuente: elaboración propia. 
TABLA 2

Ejemplos de preguntas para encuesta

\begin{tabular}{l}
\hline \multicolumn{1}{c}{ Preguntas de encuesta } \\
\hline ¿Cuántas personas viven en la casa? \\
¿Cuántos son niños? \\
¿Cuántos son adultos mayores (ancianos)? \\
¿Tiene servicio de agua potable? \\
Si tiene servicio de agua potable, ¿quién lo provee? \\
¿Qué institución regula el manejo del agua que usted consume? \\
Si no tiene el servicio de agua potable, ¿cuál es su fuente de agua? \\
En promedio, ¿cuántos días a la semana dispone de agua? \\
¿Reutiliza el agua? \\
¿Hay contaminación en el agua? ¿Qué tipo de contaminación? \\
¿Hay algún tipo de organización/institución local del agua? ¿Qué actividades realiza? \\
¿Hay un comité de cuencas? \\
¿Qué eventos extremos debidos al clima son comunes en esta zona? ¿Cuándo fue la última vez que ocurrió? \\
¿Usted pertenece a alguna organización de la colonia/comunidad? \\
¿Existe alguna sede de Protección Civil en la comunidad? \\
¿Las condiciones del suelo han cambiado con el tiempo debido a estos eventos? ¿En qué? Cuénteme un poco. \\
¿Las condiciones del agua han cambiado con el tiempo debido a estos eventos? ¿En qué? Cuénteme un poco. \\
¿Las condiciones de la vegetación han cambiado con el tiempo debido a estos eventos? ¿En qué? Cuénteme un poco. \\
¿Las condiciones de los animales han cambiado con el tiempo debido a estos eventos? ¿En qué? Cuénteme un poco. \\
\hline
\end{tabular}

Fuente: elaboración propia.

Nota: se presenta una selección de las 60 preguntas aplicadas.

\section{4. Selección de la muestra y recopilación de información en campo}

Una vez diseñado el consentimiento informado y el protocolo de encuesta, se procedió a su aplicación. El proceso se realizó durante dos semanas en la zona urbana de La Paz y en la localidad Centenario, ubicada al oeste de la ciudad. El tamaño de la muestra se definió en función del número total de Áreas Geoestadísticas Básicas (AGEBS) proporcionadas por el INEGI (2010b), las cuales son áreas urbanas conformadas por no más de 50 manzanas. Para determinar el tamaño de la muestra se aplicó la fórmula de Murray y Larry (2005):

$$
n=\frac{Z_{\alpha}^{2} \cdot N \cdot p \cdot q}{i^{2}(N-1)+Z_{\alpha}^{2} \cdot p \cdot q}
$$

En donde:

$n=$ tamaño de la muestra

$N=$ tamaño de la población $(\mathrm{AGEBS}=185)$ 
$Z=$ valor correspondiente a la distribución de Gauss (1.96)

$P=$ prevalencia esperada del parámetro a evaluar $(P=0.5)$

$q=1-\mathrm{P}$

$i=$ error que se prevé cometer $(10 \%)$

Lo anterior arrojó un resultado de 64 encuestas. Con el fin de facilitar la recopilación en campo y sistematizar su análisis, se utilizó la aplicación Epicollect + (BETA) instalada en un teléfono Android, que permitió cargar la información a la "nube" para su posterior procesamiento.

\section{5. Procesamiento de información: cálculo de índices}

Los datos recolectados se procesaron en la paquetería Excel y el cálculo de los índices se realizó con el software InfoStat versión 2008 (Di Rienzo et al., 2016). Para la construcción del índice vulnerabilidad global, se definieron una serie de rangos para determinar los niveles de vulnerabilidad: alta (1), media (0.5) y baja (0), así como las ponderaciones que son resultado de las encuestas en línea (tabla 3).

Posteriormente, se calculó el índice por cada tipo de vulnerabilidad; para ello, fue necesario homogenizar los indicadores que conforman cada capital en dicotómicas o categorizadas, que van siempre de 0 a 1 , donde 0 es la vulnerabilidad más baja y 1 la más alta (tabla 4).

Una vez homogenizadas, se multiplicaron por sus respectivas ponderaciones asignadas por los actores clave (ecuación 2) (cuadro 1), y se reescalaron los valores totales para llevarlos de nuevo a valores entre 0 y 1 y mantener la homogenización en los cálculos. Después, se sumaron los valores de los indicadores para cada capital y se calcularon los valores de cada índice; este resultado se reescaló (cuadro 2) para llevar nuevamente a valores entre 0 y 1 y obtener así el valor del índice para cada tipo de vulnerabilidad.

$$
I V H=\text { valor de la variable*ponderación }
$$

En donde:

$I V H$ : índice de vulnerabilidad humana

Valor de la variable: de 0 a 1

Ponderación: 1,2 o 3

Para obtener el índice global, se realizó el mismo procedimiento, el cual sumó los siete valores de los índices (ecuación 3), uno por cada tipo de vulnerabilidad y se reescalaron sus valores de 0 a 7 , a valores entre 0 y 1 .

$$
I V G=I V H+I V S+I V P+I V F N+I V F S+I V N+I V C
$$

En donde:

$I V G$ : vulnerabilidad global

$I V H$ : vulnerabilidad humana

$I V S$ : vulnerabilidad social

$I V P$ : vulnerabilidad política

$I V F N$ : vulnerabilidad financiera

IVFS: vulnerabilidad social

$I V N$ : vulnerabilidad natural

$I V C$ : vulnerabilidad cultural 
TABLA 3

Ponderaciones por indicador

\begin{tabular}{|c|c|c|}
\hline Vulnerabilidad/Capital & Indicador & Ponderación [2] \\
\hline \multirow{9}{*}{$\begin{array}{l}\text { Humano } \\
\text { (IVH) }\end{array}$} & 1. Población con servicio de agua & 3 \\
\hline & 2. Densidad poblacional (número de habitantes por casa) & 2 \\
\hline & 3. Enfermedades asociadas al consumo de agua & 3 \\
\hline & 4. Enfermedades asociadas a la sequía & 3 \\
\hline & 5. Enfermedades asociadas a la escasez de agua & 3 \\
\hline & 6. Conocimiento sobre acciones frente a un evento climático & 2 \\
\hline & 7. Migración & 2 \\
\hline & 8. Inmigración & 2 \\
\hline & 9. Asistencia a capacitación sobre eventos climáticos & 2 \\
\hline \multirow{5}{*}{$\begin{array}{l}\text { Social } \\
\text { (IVS) }\end{array}$} & 10. Pertenencia a organizaciones de la comunidad & 2 \\
\hline & 11. Existencia de organizaciones en la comunidad & 2 \\
\hline & 12. Percepción sobre el nivel de organización en la comunidad & 3 \\
\hline & 13. Existencia de redes de apoyo en la comunidad & 3 \\
\hline & 14. Participación en la formulación de planes & 3 \\
\hline \multirow{6}{*}{$\begin{array}{l}\text { Político } \\
\text { (IVP) }\end{array}$} & 15. Gestión de las autoridades locales & 3 \\
\hline & 16. Respuesta del gobierno (local) & 3 \\
\hline & 17. Presencia de instituciones a nivel local & 3 \\
\hline & 18. Capacidad de reacción de protección civil u otro tipo de apoyo & 2 \\
\hline & 19. Regulación del agua & 3 \\
\hline & 20. Comité de cuencas & 3 \\
\hline \multirow{6}{*}{$\begin{array}{l}\text { Financiero } \\
\text { (IVFN) }\end{array}$} & 21. Acceso a programas de gobierno & 3 \\
\hline & 22. Acceso a remesas & 2 \\
\hline & 23. Acceso a crédito & 3 \\
\hline & 24. Cambio de ingresos por evento climático & 3 \\
\hline & 25. Costo del servicio & 3 \\
\hline & 26. Costo por falta de agua & 3 \\
\hline \multirow{4}{*}{$\begin{array}{l}\text { Físico } \\
\text { (IVFS) }\end{array}$} & 27. Disponibilidad del servicio & 3 \\
\hline & 28. Infraestructura usada en caso de evento climático (albergues) & 3 \\
\hline & 29. Infraestructura afectada en caso de evento climático & 3 \\
\hline & 30. Fuentes de abastecimiento & 3 \\
\hline \multirow{7}{*}{$\begin{array}{l}\text { Natural } \\
\text { (IVN) }\end{array}$} & 31. Percepción de la vulnerabilidad del suelo & 3 \\
\hline & 32. Percepción de la vulnerabilidad del agua & 3 \\
\hline & 33. Percepción de la vulnerabilidad de la vegetación & 3 \\
\hline & 34. Percepción de la vulnerabilidad de los animales & 3 \\
\hline & 35. Percepción de cambios en el clima & 2 \\
\hline & 36. Contaminación del agua & 3 \\
\hline & 37. Recurrencia de eventos climáticos & 3 \\
\hline \multirow{6}{*}{$\begin{array}{l}\text { Cultural } \\
\text { (IVC) }\end{array}$} & 38. Comunicación ante un evento climático & 3 \\
\hline & 39. Usos del agua & 3 \\
\hline & 40. Reúso del agua & 3 \\
\hline & 41. Recurrencia a autoridades por falta de agua & 2 \\
\hline & 42. Cambios en la forma de vida & 3 \\
\hline & Total & 64 \\
\hline
\end{tabular}

Fuente: elaboración propia. 
TABLA 4

Ejemplo homogenización

\begin{tabular}{|llc}
\hline Indicador & \multicolumn{1}{c}{ Pregunta } & \multicolumn{1}{c}{ Respuesta-ponderación $[3]$} \\
\hline Población con servicio de agua potable & ¿Tiene servicio de agua potable? & Sí (0), No (1) \\
Disponibilidad del servicio de agua ${ }^{[4]}$ & $\begin{array}{l}\text { En promedio, ¿cuántos días a la } \\
\text { semana dispone de agua? }\end{array}$ & 7 días (0), 6-4 días (0.5), 3-0(1) \\
\hline
\end{tabular}

\section{CUADRO 1}

Asignación de ponderación de actores clave (ecuación 2)

\begin{tabular}{|lllc}
\hline Indicador & \multicolumn{1}{c}{ Pregunta } & \multicolumn{1}{c}{ Respuesta-Ponderación } & Ponderación actor clave \\
\hline $\begin{array}{l}\text { Población con servicio de } \\
\text { agua potable }\end{array}$ & $\begin{array}{l}\text { ¿Tiene servicio de agua } \\
\text { potable? }\end{array}$ & Sí (0), No (1) & $1 * 3=3$ \\
$\begin{array}{l}\text { Disponibilidad del } \\
\text { servicio de agua }\end{array}$ & $\begin{array}{l}\text { En promedio, ¿cuántos } \\
\text { días a la semana dis- } \\
\text { pone de agua? }\end{array}$ & 7 días (0), 6-4 días (0.5), 3-0 (1) & $0 * 3=0$ \\
\hline
\end{tabular}

Fuente: elaboración propia.

Nota: este proceso se realiza para los 64 valores de cada encuesta.

\section{CUADRO 2}

Reescala por tipo de vulnerabilidad

\begin{tabular}{lccc}
\hline Índice & Número de indicadores & Valor máximo posible & Reescala \\
\hline Humano & 9 & 0 a 9 & \\
Social & 5 & 0 a 5 & \\
Político & 6 & 0 a 6 & \\
Financiero & 6 & 0 a 6 & 0 a 1 \\
Físico & 4 & 0 a 4 & \\
Natural & 7 & 0 a 7 & \\
Cultural & 5 & 0 a 5 & \\
\hline
\end{tabular}

Fuente: elaboración propia.

Para obtener un solo valor por cada índice, se sumaron los valores de cada una de las 64 respuestas, las cuales se moverían en un rango de 0 a 64, el cual considera que el valor máximo de cada una de las 64 respuestas es 1; con esto, se genera un rango de vulnerabilidad comparable de forma general: baja (0 a 21), media (22 a 43) y alta (44 a 64).

$$
\text { Valor del rango }=\frac{\text { Valor máximo }- \text { valor mínimo }}{\text { Numero de rangos de vulnerabilidad }}
$$

En donde:

Valor máximo: 64

Valor mínimo: 0

Numero de rangos de vulnerabilidad: 3 (baja, media y alta) 
Una vez obtenido el índice de vulnerabilidad global, se procede a calcular el índice de aridez que figura como componente climático de la vulnerabilidad.

Este índice de aridez se calculó utilizando el índice de Martonne (Mercado Mancera et al., 2010; Troyo Diéguez et al., 2015) (cuadro 3), que toma como variables de estudio la precipitación y temperatura (ecuación 4).

\section{CUADRO 3}

Índice de aridez de Martonne

\begin{tabular}{|lc}
\hline \multicolumn{2}{c}{ Índice de Martonne (1926) } \\
\hline Clasificación IM & Intervalo \\
Desierto (Hiperárido) & 0 a 5 \\
Semidesierto (árido) & 5 a 10 \\
Semiárido de tipo mediterráneo & 10 a 20 \\
Subhúmeda & 20 a 30 \\
Húmeda & 30 a 60 \\
Perhúmeda & $>60$ \\
\hline \multicolumn{2}{c}{ Fuente: Mercado Mancera etal. (2010). } \\
\hline
\end{tabular}

Para obtener el índice de aridez se descargaron Grids con datos de precipitación y temperatura de la página WorldClim [5] de 1950 al 2000. Los Grids se procesaron en ArcGIS versión 10.2 mediante la herramienta extraction-multipoint para obtener valores de $P p$ y $T$ correspondientes a cada punto encuestado.

$$
A=\frac{P p * 12}{T+10}
$$

En donde:

$A=$ Índice de aridez

$P=$ Precipitación mensual en $\mathrm{mm}$

$T=$ Temperatura mensual en ${ }^{\circ} \mathrm{C}$

$12=$ Constante que considera los meses del año

$10=$ Constante adimensional de ajuste de escala

Los resultados del índice de Martonne se reescalaron, en este caso, reversando el código de la escala de 0 a 1 (cuadro 4) para posteriormente multiplicarlo por el valor del índice global, reescalarlo y así obtener el índice de vulnerabilidad integral (Aguilar, 2007).

\section{CUADRO 4}

Reclasificación índice de Martonne

\begin{tabular}{|lc}
\hline \multicolumn{2}{c}{ Reclasificación índice de Martonne } \\
\hline Clasificación IM & Valor reescalado \\
\hline Desierto (Hiperárido) & 1 \\
Semidesierto (árido) & 0.8 \\
Semiárido de tipo mediterráneo & 0.6 \\
Subhúmeda & 0.4 \\
Húmeda & 0.2 \\
Perhúmeda & 0 \\
\hline
\end{tabular}

Fuente: elaboración propia basada en Mercado Mancera et al. (2010). 
Una vez obtenido el índice de aridez, que representa al componente climático, se aplicó la siguiente fórmula para obtener el índice de vulnerabilidad integral (ecuación 6):

$$
I V I=I V G * I A M
$$

En donde:

$I V I=$ Índice de vulnerabilidad integral

$I V G=$ Índice de vulnerabilidad global

$I A M=$ Índice de aridez de Martonne

\section{Mapas de Vulnerabilidad}

Para hacer uso correcto de los datos calculados para cada índice, se realizó una estandarización de ellos con la transformación raíz del ArcoSeno en InfoStat (Balzarini et al., 2008). Esta transformación permite que las varianzas sean constantes en toda el área de estudio y que los datos se distribuyan normalmente (ESRI, 2014).

$$
\operatorname{ArcoSeno}(\operatorname{Raíz}(p))
$$

Se procesó la capa de datos de la transformación en ArcGis versión 10.2 mediante la herramienta de interpolación Inverse Distance Weighted (IDW); de este cálculo se obtuvo un archivo en formato ráster al que posteriormente se le aplica el inverso de la transformación de ArcoSeno:

$$
\operatorname{Sen}^{-1}(\sqrt{p})
$$

Una vez obtenidos los datos de esta conversión, se procedió a diseñar los mapas de distribución de los valores asociados a los diferentes tipos de vulnerabilidad en la zona de estudio.

\section{Resultados y Discusión}

Las principales diferencias entre esta metodología y otras que se han propuesto para el análisis de vulnerabilidad consisten en la forma de integrar los datos con sus correspondientes cálculos. Por ejemplo, en algunos casos se utilizan promedios ponderados de los valores asignados a cada variable para el cálculo de vulnerabilidad (Ríos et al., 2011). En el caso de Bouroncle et al. (2016), para calcular índices de capacidad adaptativa, se hace uso sólo de información de bases de datos tipo censo y repositorios de información biofísica sin tomar en cuenta la percepción social. En cuanto a esta propuesta, se realizó una suma ponderada para evitar sesgo en los datos y se atendió en especial la percepción de la comunidad.

En otras investigaciones la vulnerabilidad está enfocada en los elementos individuales de carácter social, físico o ambiental, los cuales se analizan por separado (Álvarez, 2014; Soares y Gutierréz-Montes, 2011). En lo que corresponde a este artículo, la vulnerabilidad es considerada un sistema integrado por siete subsistemas: humano, social, político, financiero, físico, natural y cultural (Gutierréz-Montes et al., 2014).

El MCC se ha aplicado además en Estados Unidos de América y Uganda para buscar el aprovechamiento de capitales de la comunidad y la mejora de los medios de vida (Flora y Harris Gillespie, 2009; Sseguya et al., 2009). Por lo tanto, se considera que la vulnerabilidad debe ser medida de forma integral, de manera que se analicen todos los sectores/grupos/elementos que se conecten al objeto de estudio (Lampis, 2013; Merrey et al., 2005). 
Los índices de vulnerabilidad calculados y espacializados permiten ver la desigualdad en la distribución no nada más de agua potable en La Paz sino de otros recursos. Se dice que al no hacerse de manera uniforme la disponibilidad del agua en la población, en conjunto con una inadecuada capacidad socioeconómica de tratar esto, deja a un gran número de personas sin poder acceder a fuentes de agua, por lo que se presentan crisis a diferentes escalas (Pandey et al., 2011). Asimismo, el crecimiento urbano y el desarrollo de la ciudad pueden acentuar aún más los efectos de esta desigualdad, a lo que se añade la baja calidad del agua, degradación de otros recursos (flora, fauna, infraestructura) y efectos adversos en caso de inundaciones.

La vulnerabilidad de toda la zona de estudio fue media. El valor más alto se presentó en la humana (49.48). Respecto a la social (43.33), política (39.50), financiera (38.51), natural (41.38) y cultural (39.29), éstas cuentan con valores medios. Para la física se obtuvo un valor de (24.11), el más bajo de los valores obtenidos, pero aún encontrándose en un rango medio. Tanto el índice global como el integral cuentan con un valor de 34.96, lo que indica además de una vulnerabilidad media, que la vulnerabilidad global de la zona no se ve afectada significativamente por el índice climático calculado (cuadro 5).

CUADRO 5

Resultado de índices de vulnerabilidad por capital

\begin{tabular}{|lcc}
\hline \multicolumn{1}{c}{ Índice } & Valor del índice & Nivel de vulnerabilidad \\
1. Humano & 49.48 & Alta \\
2. Social & 43.33 & Media \\
3. Político & 39.50 & Media \\
4. Financiero & 38.51 & Media \\
5. Físico & 24.11 & Media \\
6. Natural & 41.38 & Media \\
7. Cultural & 39.29 & Media \\
8. Global & 34.96 & Media \\
9. Integral & 34.96 & Media \\
\hline
\end{tabular}

Fuente: elaboración propia.

A continuación, se presenta un análisis de los resultados obtenidos por cada índice de vulnerabilidad. En los mapas explicativos se podrá observar una semaforización: el color rojo representa vulnerabilidad alta, el amarillo vulnerabilidad media y el verde vulnerabilidad baja.

\section{1. Vulnerabilidad humana}

El índice de vulnerabilidad humana fue alto, pues obtuvo 49.48 puntos de 64, que parece estar dado en gran manera por la baja asistencia de las personas a capacitación. De los encuestados, $12 \%$ asiste a eventos de capacitación; este porcentaje comenta que las charlas sólo se enfocan en ahorrar agua y no se tocan otros temas. El resto (88\%) no tiene tiempo, no le interesa o no se entera. De cualquier manera, no hay charlas o la gente no asiste porque no se llevan a cabo. De los encuestados, 95\% cuenta con servicio de agua potable o entubada en sus domicilios, y 6\% de los casos se asocia a la sequía y escasez de agua, enfermedades como diarrea, malestar estomacal y deshidratación. En general, en el mapa 2 predomina el color amarillo con leves tintes rojos y en la parte noreste se observa una mancha roja más marcada. Esta zona es pobre, con menos servicios, menos oportunidad de crear centros de capacitación si se compara con la zona céntrica de la ciudad, la cual cuenta con más servicios y movimientos económicos importantes; esto deriva en un bajo conocimiento de toda la comunidad respecto a acciones ante eventos climáticos extremos, acentuado en las zonas pobres. 


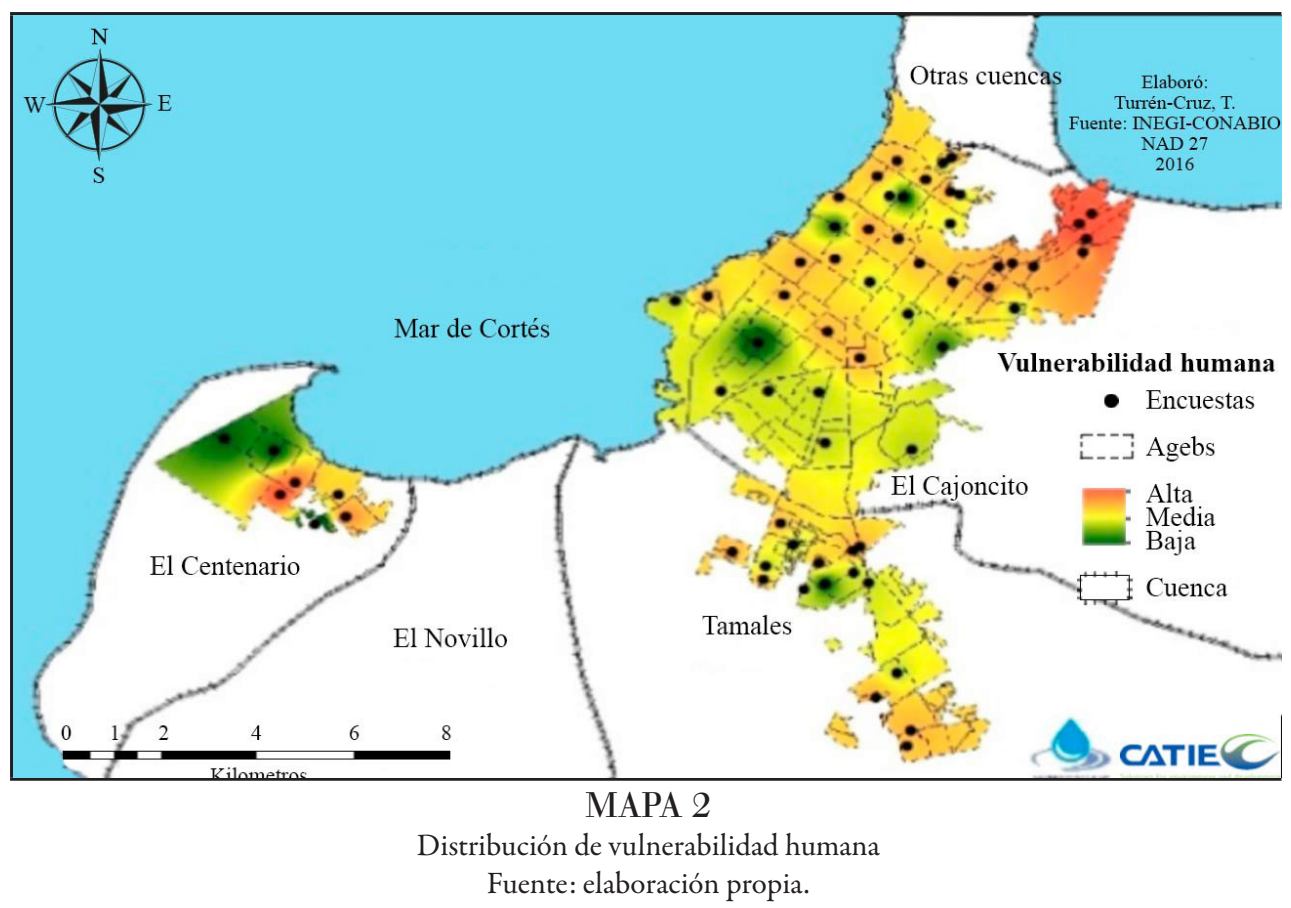

En 2003 se dijo que alrededor de 14 millones de personas en Etiopía estaban en peligro de inanición debido a la pérdida de cultivos por la sequía. Para el periodo 2012-2013, Guatemala tuvo malas cosechas por este mismo motivo provocando la reducción de empleo y desnutrición en niños. En septiembre de 2014 en Nicaragua, Honduras y El Salvador, más de 500000 familias no tenían qué comer debido a la sequía. Estos y otros eventos devastadores del mundo que vemos siempre en las noticias son una parte de la historia a la cual la pobreza está ligada (Flores Mora, 2014; Merrey et al., 2005). En la zona de Centenario (suroeste) se puede observar una mancha verde más amplia; en ella se dan mayor cantidad de "capacitaciones", pero debidas principalmente a actividades asociadas a las escuelas primaria y secundaria, en su mayoría obligatorias.

La baja asistencia a actividades de participación puede definirse por la falta de interés tanto de la comunidad como de las autoridades. Hay una brecha comunicacional y de confianza en la que la comunidad ya no confía en las autoridades debido a que al parecer no han hecho lo posible por mejorar esta percepción y relación con la comunidad, lo cual debilitó su capital humano, y disminuye así los conocimientos y destrezas de la comunidad. Esto implica la necesidad de capacitaciones con cierta periodicidad cubriendo más temas como el reúso del agua, qué hacer en caso de huracanes o tormentas, cómo afrontar los efectos de la sequía y la escasez, además de aprovechar estos espacios para crear un canal de flujo de información, conocer las necesidades de los habitantes y diseñar planes de acción para dar soluciones. Cruz Falcón et al. (2009) sugieren, entre otras actividades, implementar programas sobre la cultura del agua y un plan de manejo específico, ya que este recurso no es valorado.

\section{2. Vulnerabilidad social}

El índice de vulnerabilidad social fue medio, el cual se encuentra en el límite del rango con 43.33 puntos de 64. Este índice parece determinado por la baja presencia de organizaciones en la comunidad, únicamente en $5 \%$ de los casos hay presencia de organizaciones sociales que, por lo general, son comités locales que surgen cuando hay problemas urgentes que atender como la falta de luz. Además, hay escasa presencia de redes locales de apoyo: solamente $19 \%$ de los casos cuenta con un "enlace" que permite comunicar 
a la comunidad con el gobierno (estos son los jefes o jefas de colonia), pero posee credibilidad, ya que las personas dicen que "busca su propio beneficio" porque primero buscan satisfacer sus necesidades, después las de sus allegados y al final las del resto de la comunidad. A esto, se suma la baja participación para formular planes de acción, lo cual se ve ligado a la vulnerabilidad del capital humano con la baja asistencia a capacitación y la falta de interés comunidad-autoridades, que a su vez disminuye su capacidad de respuesta para enfrentar y recuperarse ante un evento. Las soluciones que permiten la capacidad adaptativa requieren del soporte de la organización social como grupos civiles y cooperativas locales, entre otros, con el fin de que la comunidad trabaje en conjunto para enfrentar retos y esté más organizada para poder permitir que los miembros de la comunidad tengan conocimiento y acceso a los recursos necesarios para su adaptación y ayudar al mismo tiempo al empoderamiento de la comunidad (Baca et al., 2014; Emery y Flora, 2006).

Podemos observar en el mapa 3 el color rojo sobre todo en las zonas periféricas noreste, sureste y noroeste, las cuales corresponden a áreas económicas pobres y algunas de reciente creación. Puede que en estas zonas la presencia de instituciones, la participación y la organización como comunidad se dificulte por la falta de recursos y el sentido de pertenencia.

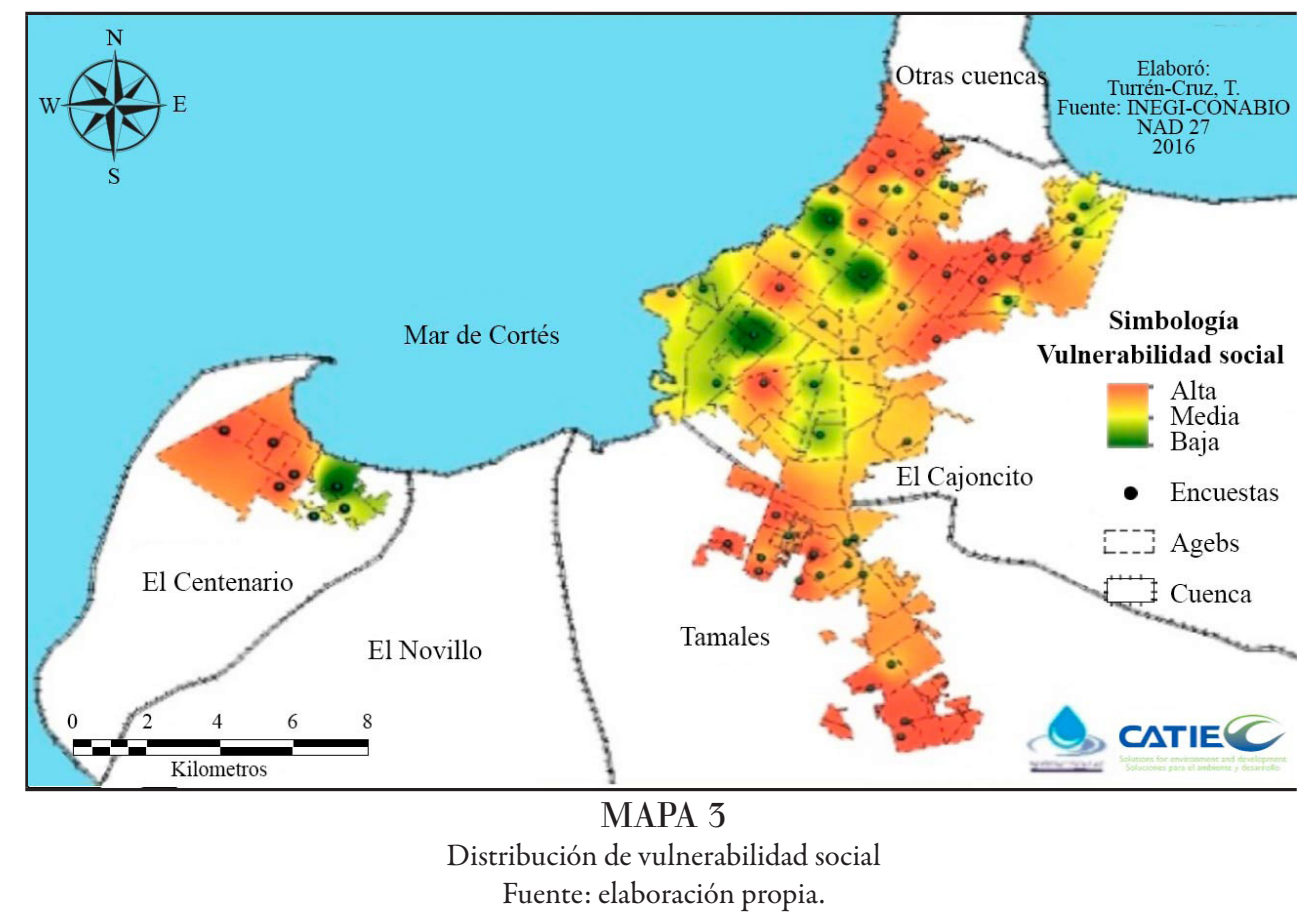

\section{3. Vulnerabilidad política}

Elíndice de vulnerabilidad política fue medio con 39.50 puntos de 64. La comunicación en caso de emergencias es buena, en las cuales se usan como medios principales de comunicación la radio, la televisión, el perifoneo local y, en casos puntuales extremos, la ayuda de protección civil y los soldados. La respuesta del gobierno es calificada como de regular a buena en las zonas rojas del mapa 4. Esta calificación corresponde a regular, lo cual se podría explicar porque estas son zonas alejadas del centro; en ellas quizá la atención de las necesidades de los habitantes no se da con tanta rapidez como se da en las céntricas, en donde los colores se perciben en su mayoría amarillo y verde reflejando una vulnerabilidad media que posiblemente indica una atención rápida a sus necesidades. Aun así, las personas comentan que las autoridades "hacen lo que pueden, intentan resolver los problemas" y que "no se dan abasto". Asociado a esto, hay carencias por estructura insuficiente, ineficiente 
e inexistente. En estas mismas zonas rojas, hay lugares en los que no se cuenta con red de distribución de agua potable, no hay tomas en las casas y el abastecimiento se hace mediante pipas que el Organismo Operador Municipal del Sistema de Agua Potable y Saneamiento de La Paz (OOMSAPAS) envía o que los pobladores compran. En otros lugares de la ciudad se ha realizado la instalación de medidores (Cruz Falcón et al., 2009) con el fin de medir el consumo en pro de mejorar el servicio, pero los pobladores comentan que debido a la ineficiente estructura de la red de agua no resultan una buena opción; aunque miden el aire que llega por las tuberías a falta de presión en el abastecimiento, no es rentable para los habitantes de la ciudad, por lo que afecta además su capital financiero.

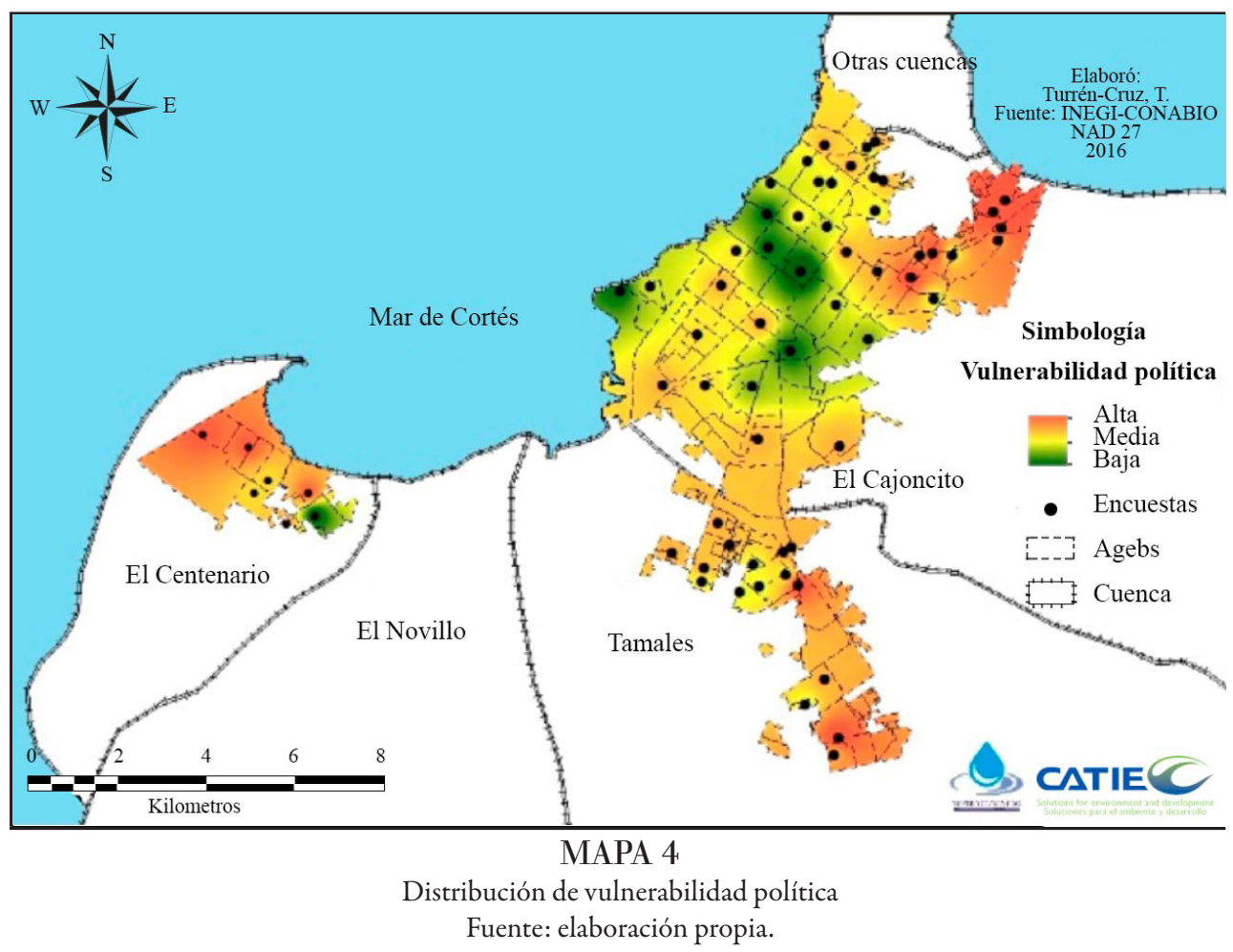

De los pobladores, entre 95 y $98 \%$ desconoce si hay un comité de cuencas, consejo de cuencas o cualquier otro tipo de organización o autoridad que pueda orientarlos o ayudarlos en la resolución de problemas y satisfacción de sus necesidades, no tienen conocimiento de a dónde o a quién pueden acudir. Esta carencia también está asociada a su capital humano y social afectando negativamente los ya de por sí limitados conocimientos, participación y relaciones de apoyo ante eventos climáticos. Sería de gran ayuda organizar un programa de entrega de pipas a las zonas necesitadas con una frecuencia establecida y dar a conocer las organizaciones o instituciones a las que se puede acudir y para qué asuntos; esto podría hacerse en los espacios de capacitación referidos. Por otro lado, Cruz Falcón y Troyo Diéguez (2010b) proponen la creación de un instituto del agua para identificar los problemas de abasto, así como buscar soluciones, realizar investigaciones, gestionar recursos hídricos y promover la capacitación de recursos humanos.

\section{4. Vulnerabilidad financiera}

El índice de vulnerabilidad financiera fue medio con 38.51 puntos de 64. De las personas consideradas, $18 \%$ cuenta con apoyo financiero que recibe cada dos meses a través de programas de gobierno como Sin hambre y 
Prospera. En el mapa 5 las manchas verdes corresponden a las zonas en donde la gente cuenta con estos apoyos. Se puede apreciar que algunos caen en zonas periféricas de bajos recursos y, al tener estos apoyos, parecen ser "menos vulnerables", lo que sucede contrariamente en las zonas centrales que se tiñen de rojo al no contar con ese recurso económico extra.

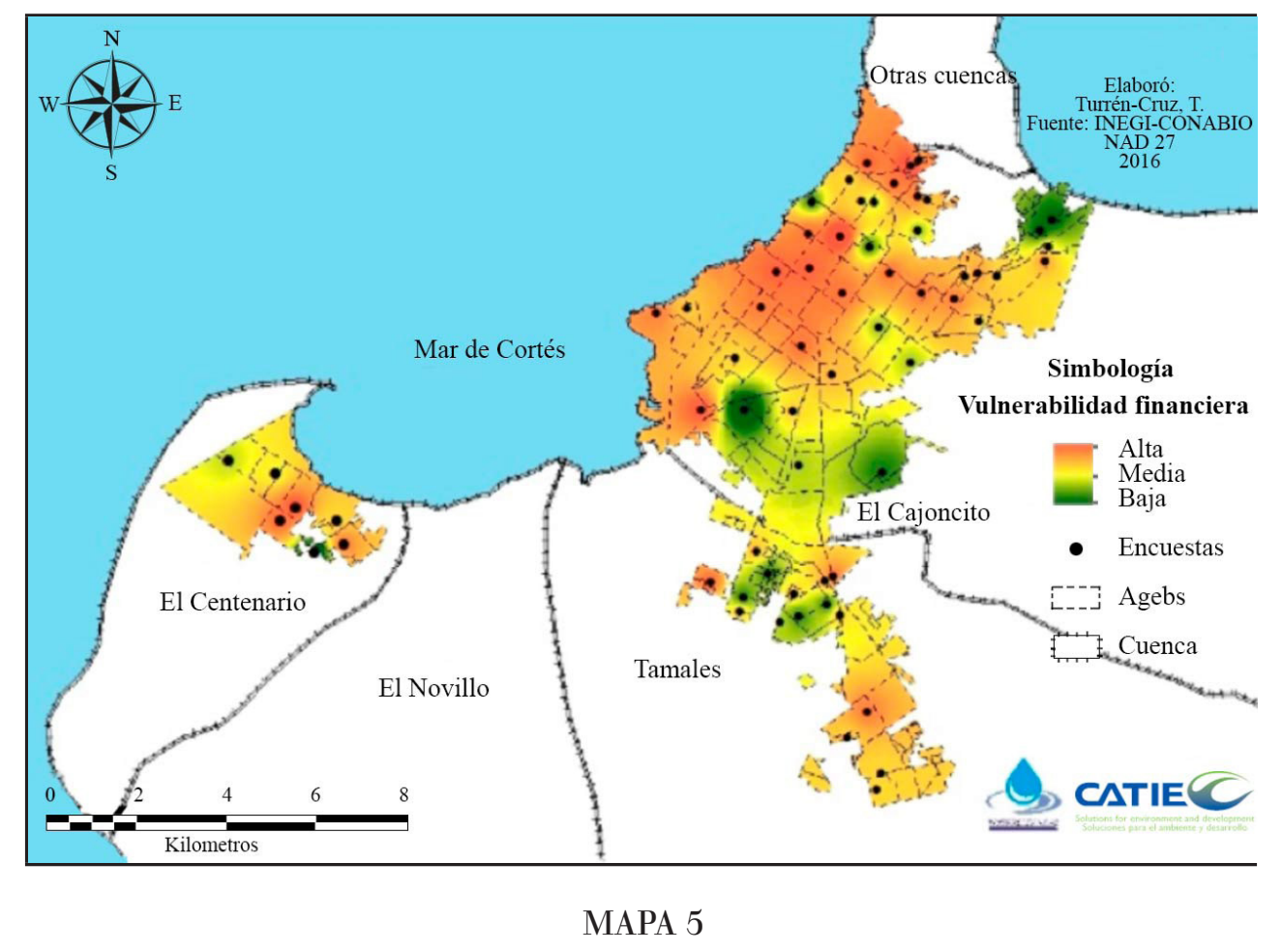

Distribución de vulnerabilidad financiera

Fuente: elaboración propia.

La Paz es una ciudad pequeña si se compara con otras urbes del país, se caracteriza por ser turística y tranquila, lo cual se refleja en que $100 \%$ de los encuestados no conoce a alguien que haya migrado al extranjero u otras ciudades del país en busca de una mejor vida a pesar de las condiciones asociadas al clima que padece la zona y tampoco hay ingresos por remesas.

De quienes poseen crédito y cuentan con uno, $42 \%$ tiene acceso a ello. Lo anterior se ve reflejado en las zonas amarillas del mapa que vuelven a ser las zonas periféricas y pobres de la ciudad en donde las personas obtienen créditos con bancos o tiendas departamentales ya sea para satisfacer necesidades inmediatas del hogar o pagar deudas (los pobres adquieren deudas para pagar deudas). En el caso de la zona céntrica, las personas tienen acceso a crédito, pero no lo adquieren por miedo a endeudarse, prefieren "ir al día". Esta situación no se da en la periferia, ya que esta zona es menos activa económicamente.

De los encuestados, $67 \%$ ha sufrido afectaciones económicas durante un evento climático extremo como daños a la vivienda que han sido menores y también debido al tipo de material (madera, cristal, lámina o mala construcción), falta de trabajo, compra de víveres, entre otros. El porcentaje de quienes tienen gastos extras por falta de agua es de 75\%; los gastos van de 100 a 400 pesos al mes.

Esta situación sustenta la necesidad de monitorear los programas de gobierno para asegurar que los recursos se usen de acuerdo con su objetivo, así como generar más facilidades para el acceso a créditos sobre todo a personas de bajos recursos. La disminución de la falta de agua reducirá gastos y permitirá a las familias usar ese recurso económico en otras necesidades fundamentales. [6] 


\section{5. Vulnerabilidad física}

El índice de vulnerabilidad física fue medio con 24.11 puntos de 64. En este capital sucede algo interesante, ya que se puede observar que el mapa 6 se tiñe en su mayoría de color verde porque se encuentra en el nivel más bajo del rango medio de la vulnerabilidad, lo que nos permite deducir que este capital posiblemente se encuentre en un proceso de transición de niveles bajos a medios de vulnerabilidad. Esta situación puede estar asociada a que sólo $73 \%$ de las personas cuentan con servicio de agua potable tres días a la semana. Esto es común que ocurra en zonas céntricas y en colonias de reciente creación como en el caso de la parte sureste de la ciudad, donde se mezclan colonias antiguas (La Fuente, El Dorado, Misiones, etcétera) con las más recientes (Camino Real, El Palmar y Ayuntamiento).

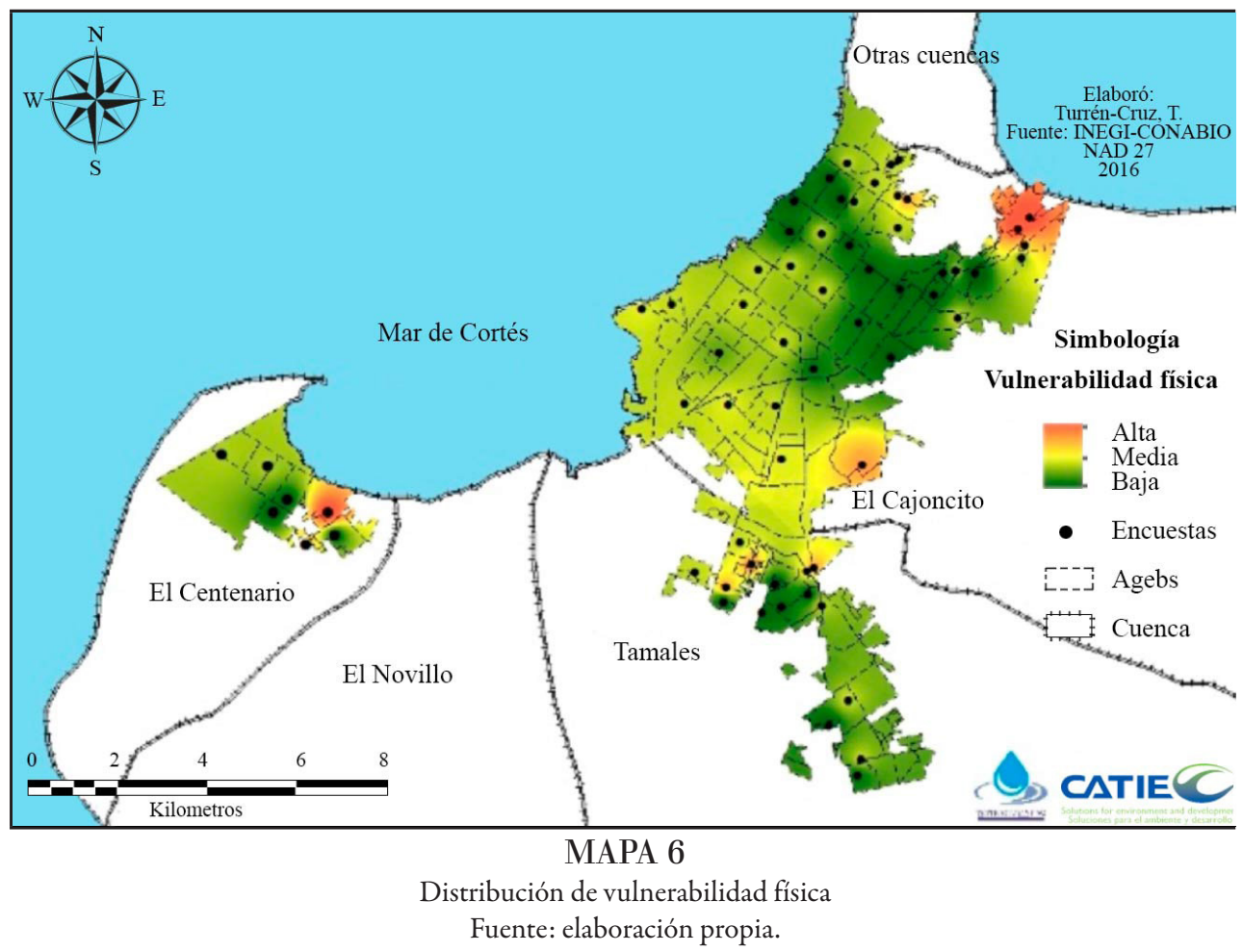

En caso de emergencias, las personas saben a qué lugar asistir, tienen albergues designados, por lo regular primarias y secundarias. Esto se relaciona mucho con el capital político en el cual, como se mencionó, las autoridades han realizado una buena gestión de comunicación.

En $41 \%$ de los casos ha habido daños a infraestructura, pero han sido menores debido al tipo de material (madera, lámina y cristal). En $100 \%$ de los casos la fuente de abastecimiento de agua es proporcionada por el OOMSAPAS, lo cual revela la necesidad de dar seguimiento a programas que dan apoyo de material para construcción a las personas con bajos recursos y así mejorar la calidad de sus viviendas y sobrellevar los impactos de eventos climáticos.

\section{6. Vulnerabilidad natural}

El índice de vulnerabilidad natural fue medio con 41.38 puntos de 64. Los encuestados en general han percibido cambios en el suelo, agua y vegetación, baja fertilidad, sequedad, escasez y pérdida de especies. En el caso de la fauna, los cambios percibidos son focalizados en el área de ganadería. Algunos encuestados comentan que "las 
vacas se enflacan y mueren, ya no hay pastura buena”. En la pesca se dio la desaparición de la almeja Catarina. Estas pérdidas de igual manera afectan el capital financiero de la comunidad, por lo tanto sus ingresos. Las personas han percibido cambios en el clima sobre todo el aumento de temperatura: "El clima está loco, cuando debería hacer frío hace calor y al revés".

En el mapa 7 las manchas rojas corresponden a la zona céntrica; en esta área las personas perciben más cambios en los recursos naturales y el clima por la mancha urbana creciente. Si esto se compara con las demás zonas, la vulnerabilidad es en su mayoría amarilla y verde con algunos puntos rojos muy focalizados, donde las personas han visto los cambios en este aspecto, puesto que se han visto afectadas de manera directa como en el caso de los pescadores por la desaparición de la almeja Catarina y los ganaderos que han perdido su vacas; en casos contrarios no han notados grandes alteraciones. Por lo anterior, se identifica la necesidad de diversificar sus actividades productivas.

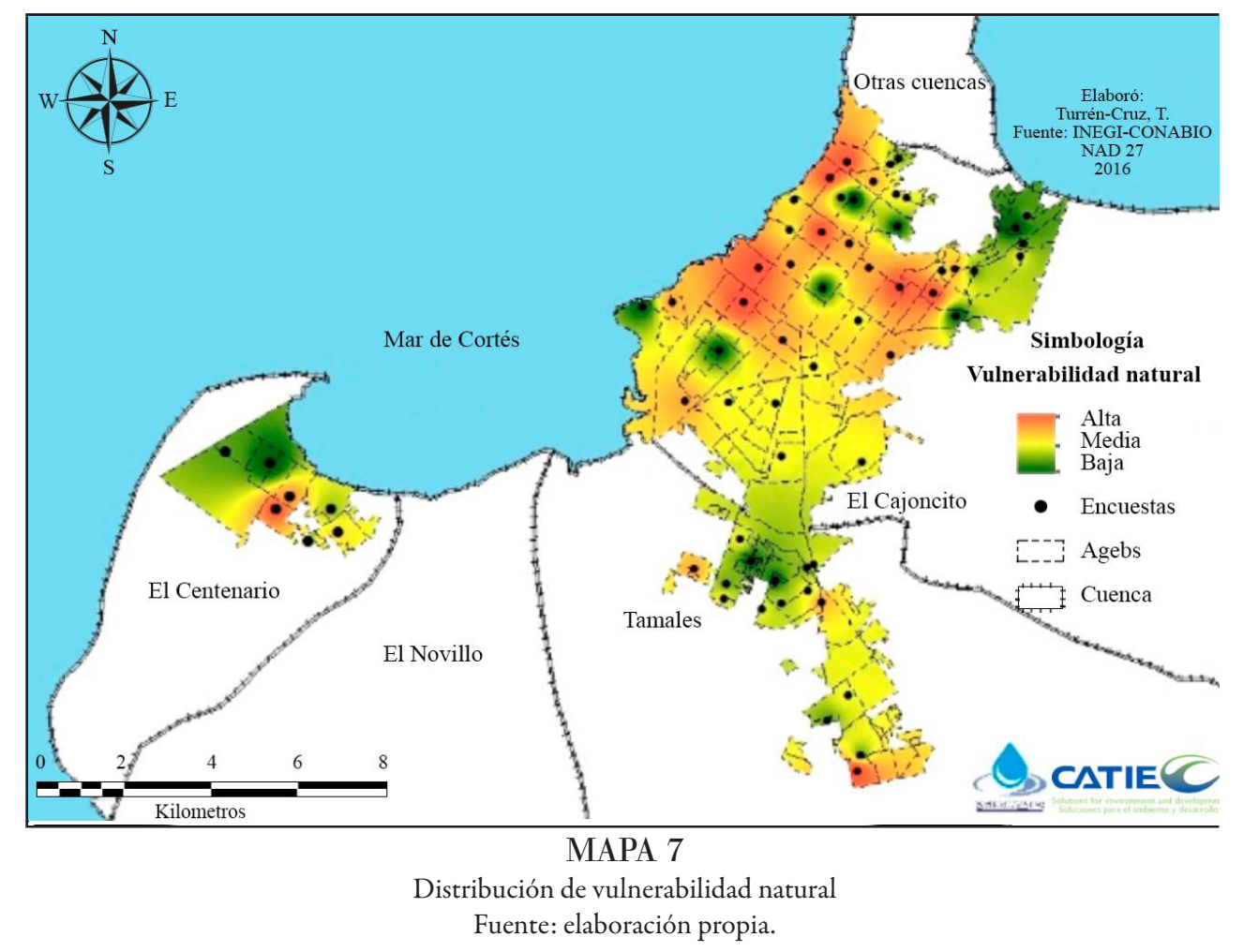

\section{7. Vulnerabilidad cultural}

El índice de vulnerabilidad cultural fue medio con 39.39 puntos de 64. Dentro de la comunidad en general se observan buenas redes de comunicación en caso de un evento climático extremo. Los principales medios de comunicación que se utilizan son la radio a través de dos canales locales $\mathrm{HZ}$ y 100.7 FM, la televisión mediante grandes televisoras como Televisa y Tv Azteca, a la par de los dos canales locales 8 y 10, y el internet por medio de las plataformas Google o Facebook. En las zonas periféricas y pobres se perciben mejores redes de comunicación, quizá asociadas a la dificultad para acceder a recursos y a su bajo nivel de vida, comparado con la zona céntrica de la ciudad en donde la comunicación entre vecinos parece ser menor, esto es observable en los puntos rojos del mapa 8, pues se puede percibir menos "necesidad" de comunicación y apoyo, dado probablemente por la mejor calidad en infraestructura y facilidad para acceder a recursos. El uso del agua es mayormente doméstico, incluso en las zonas centrales donde los negocios se dedican a la venta de souvenirs y oferta de servicios de comida, por lo que el consumo del recurso parece no ser tan elevado. En casos de falta de agua, $92 \%$ de los encuestados no 
recurre a autoridades sino a familiares o vecinos con los que cooperan para pedir pipas y satisfacer la necesidad. Es importante conocer la cultura de la comunidad respecto al recurso para saber qué conciencia tiene en cuanto a su uso; en este caso, el uso se ve relacionado directamente con el capital humano y político, en el cual la comunidad no sabe a dónde recurrir para resolver la situación o no confía en la resolución de sus problemas. El porcentaje que ha sufrido cambios en su forma de vida es de $34 \%$ debido al daño en bienes materiales, pérdida de trabajo e inversión en mejoras de la casa como la construcción de cisternas y formas de almacenamiento de agua o han tenido que dejar de invertir en necesidades básicas como educación para solventar la falta del recurso hídrico. Este elemento está muy relacionado con el capital financiero, ya que algunos de los apoyos de gobierno recibidos no se usan para el objetivo deseado sino que se invierten en este tipo de mejoras para el hogar por ejemplo.

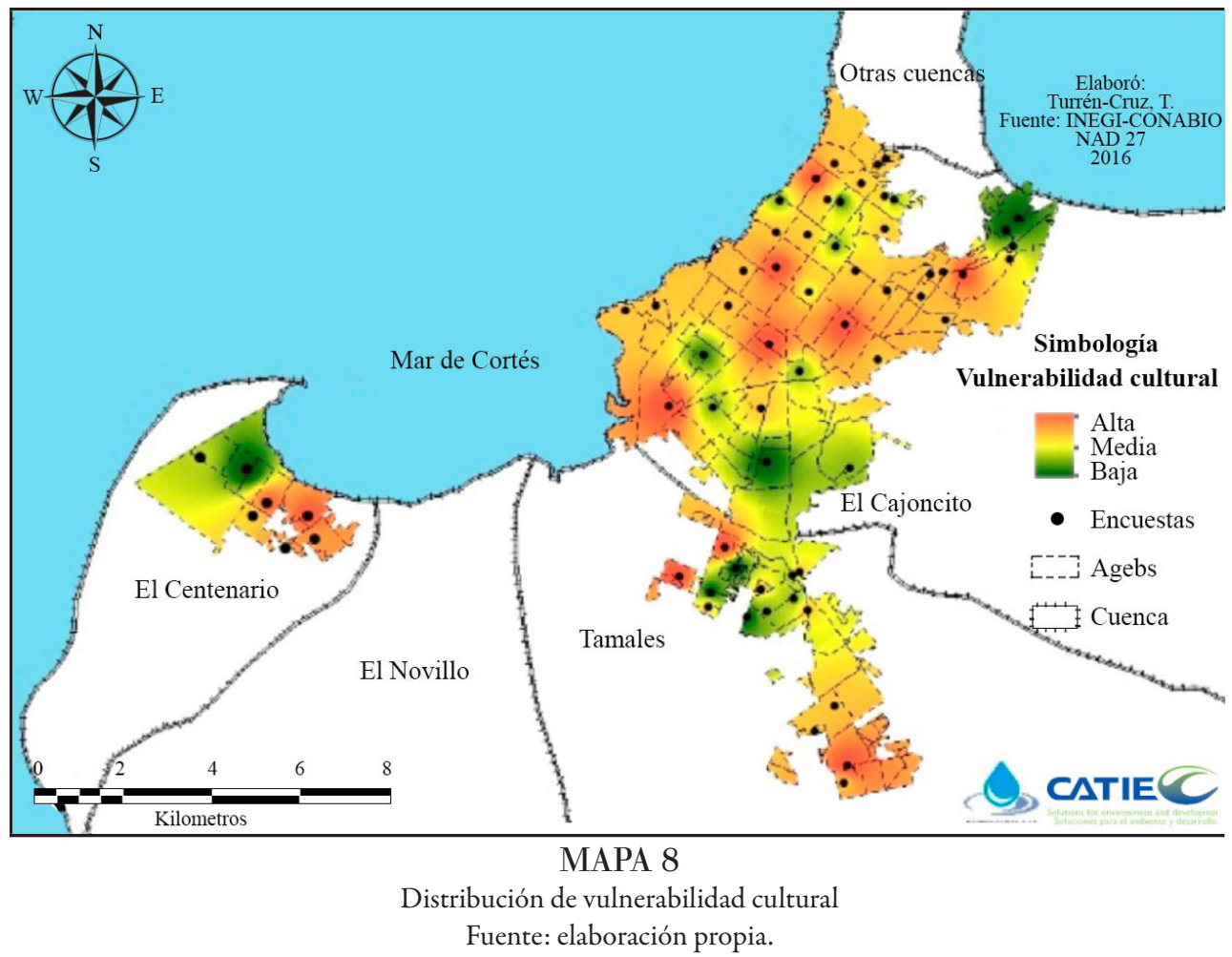

En $77 \%$ de los casos el agua no se reutiliza debido a que hay poca cultura de reúso, sobre todo en las zonas céntricas (manchas rojas) donde las personas por su mayor solvencia económica, o por ser un sector con más movimiento económico y mejor infraestructura, cuenta con cisternas o tanques de almacenamiento de agua; entonces, el recurso no hace falta casi nunca, por lo que no es indispensable reusarla e incluso la ocupan para lavar aceras y regar plantas. Lo contrario sucede en las zonas periféricas de la ciudad en el sureste y suroeste (Centenario), pues aquí el agua es un recurso valioso. Como no siempre la tienen, deben pagar extra por ella y esto afecta directamente en su capital financiero derivando en una mejor apreciación del recurso y lo usan también para lavar ropa, regar las plantas y el baño. En este aspecto, se ha propuesto diseñar estrategias para captación de lluvia en los techos de casas y edificios (Cruz Falcón y Troyo Diéguez, 2010b).

\section{8. Vulnerabilidad global e integral}

El índice global integra los índices humano, social, político, financiero, físico, natural y cultural, mientras que el índice integral está formado por el producto del índice global y un índice climático; este último tiene un valor de 0.8 correspondiente a una alta vulnerabilidad para toda la zona y se categoriza como "semidesierto (árido)". Se puede observar en el mapa 9 y 10 que no hay diferencias significativas entre la vulnerabilidad global 


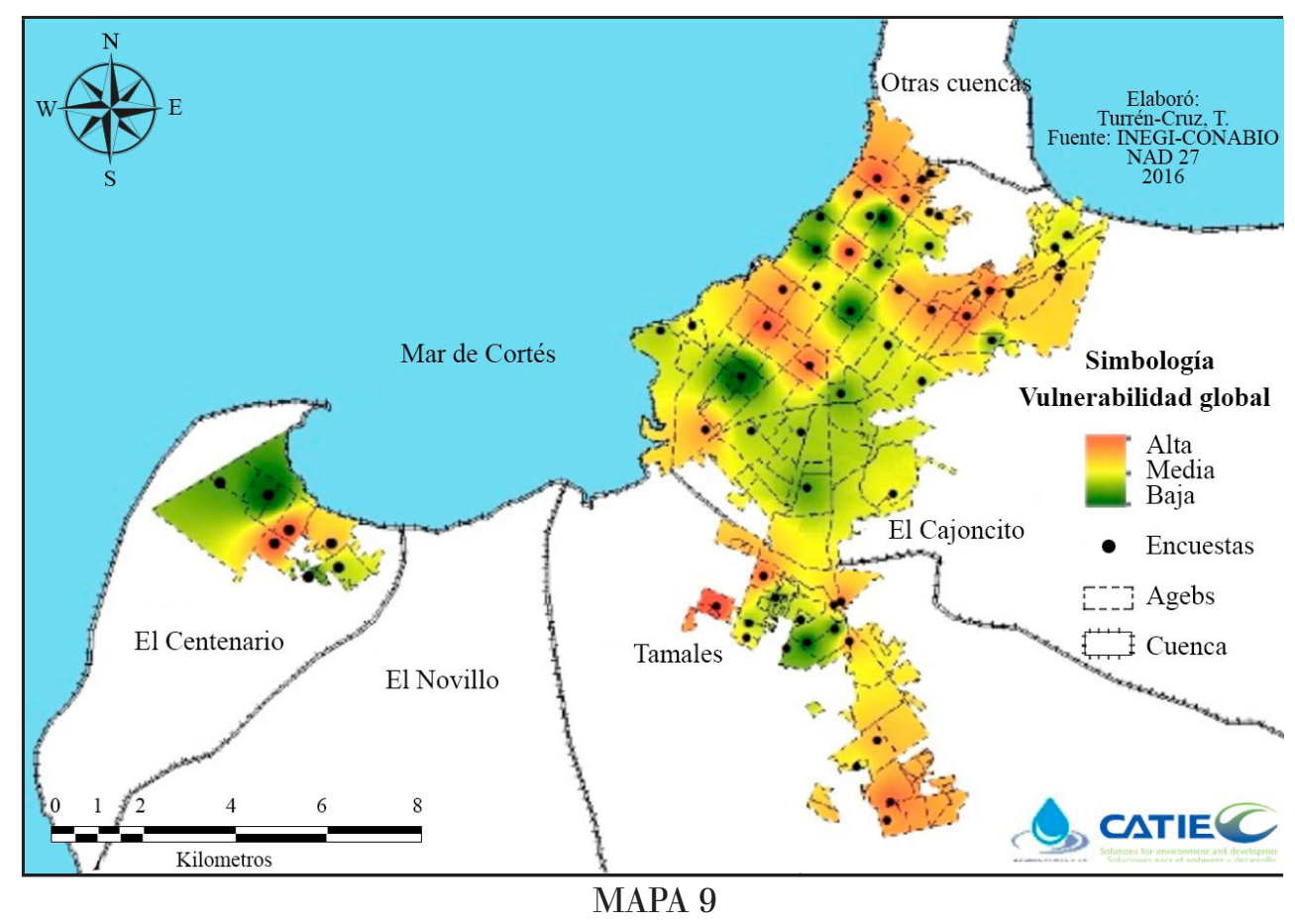

Distribución de vulnerabilidad global

Fuente: elaboración propia.

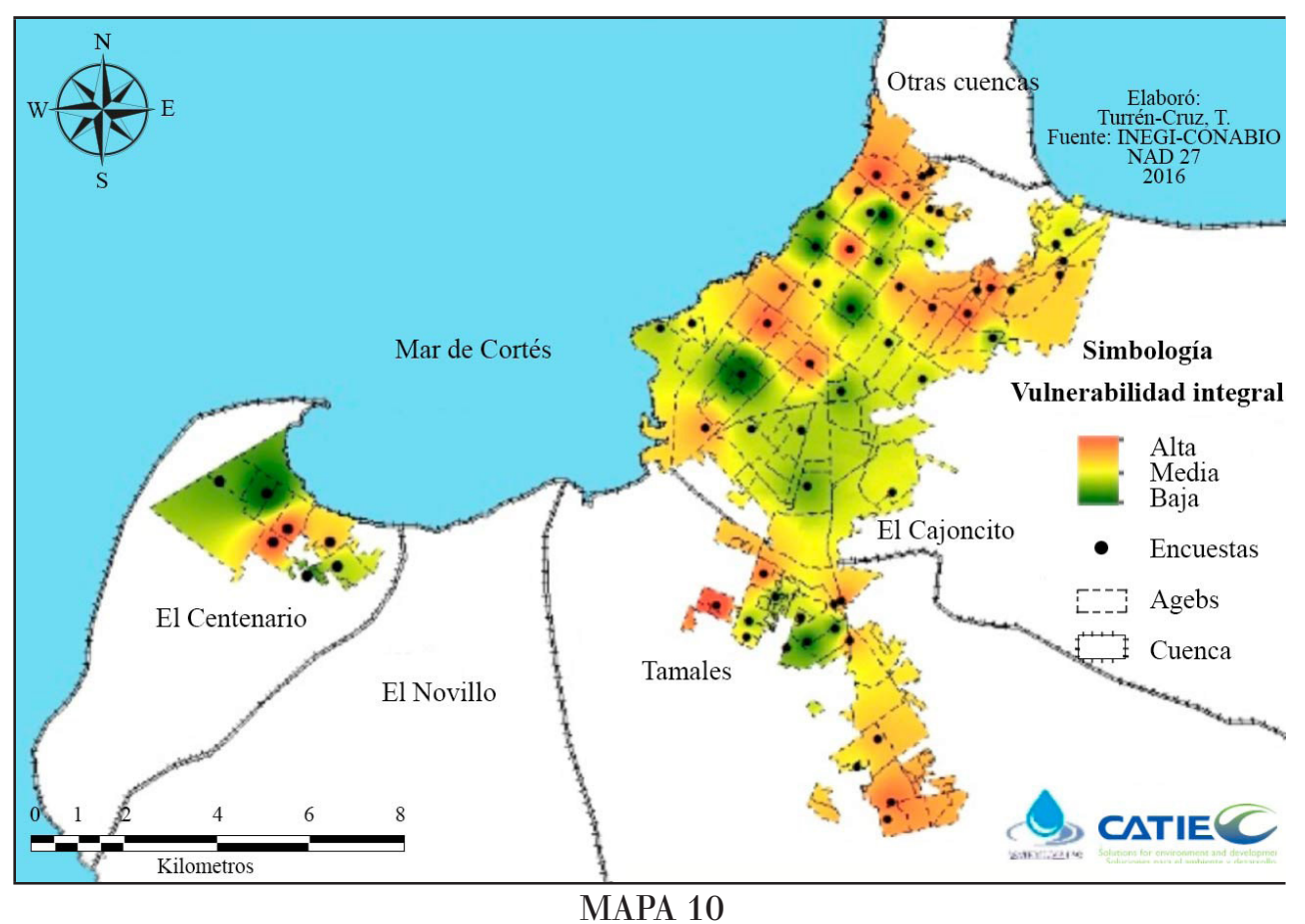

Distribución de vulnerabilidad integral

Fuente: elaboración propia.

e integral, lo cual podría reflejar que el índice climático calculado tiene poca influencia en la vulnerabilidad de la zona. Esto puede deberse a que el área estudiada es pequeña y muy homogénea. De acuerdo con datos de CONABIO (2010) e INEGI (2010a), diversas variables biofísicas como precipitación, temperatura, altura, tipo de vegetación, entre otras, no presentan variaciones importantes. 
La baja incidencia del índice climático parece estar influenciada por la homogeneidad de las características biofísicas de la zona, sobre todo de precipitación y temperatura que son las variables de interés en el cálculo del índice de Martonne. La altura tiene un rango de 0 a $200 \mathrm{msnm}$, la precipitación varía entre 100 hasta $300 \mathrm{~mm}$ y la temperatura de 22 a $24^{\circ} \mathrm{C}$ (mapa 11, mapa 12 y mapa13).

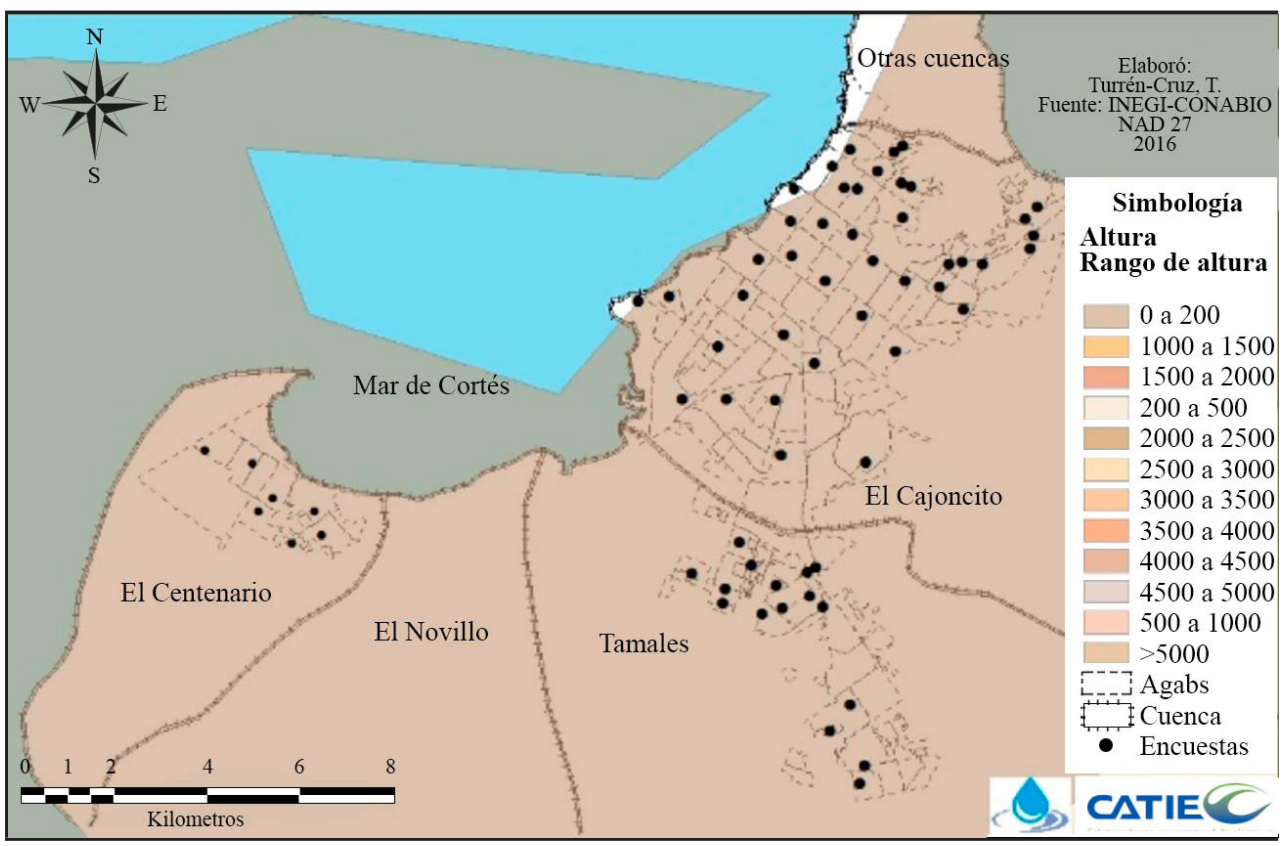

MAPA 11

Rango de altura en La Paz, BCS

Fuente: elaboración propia.

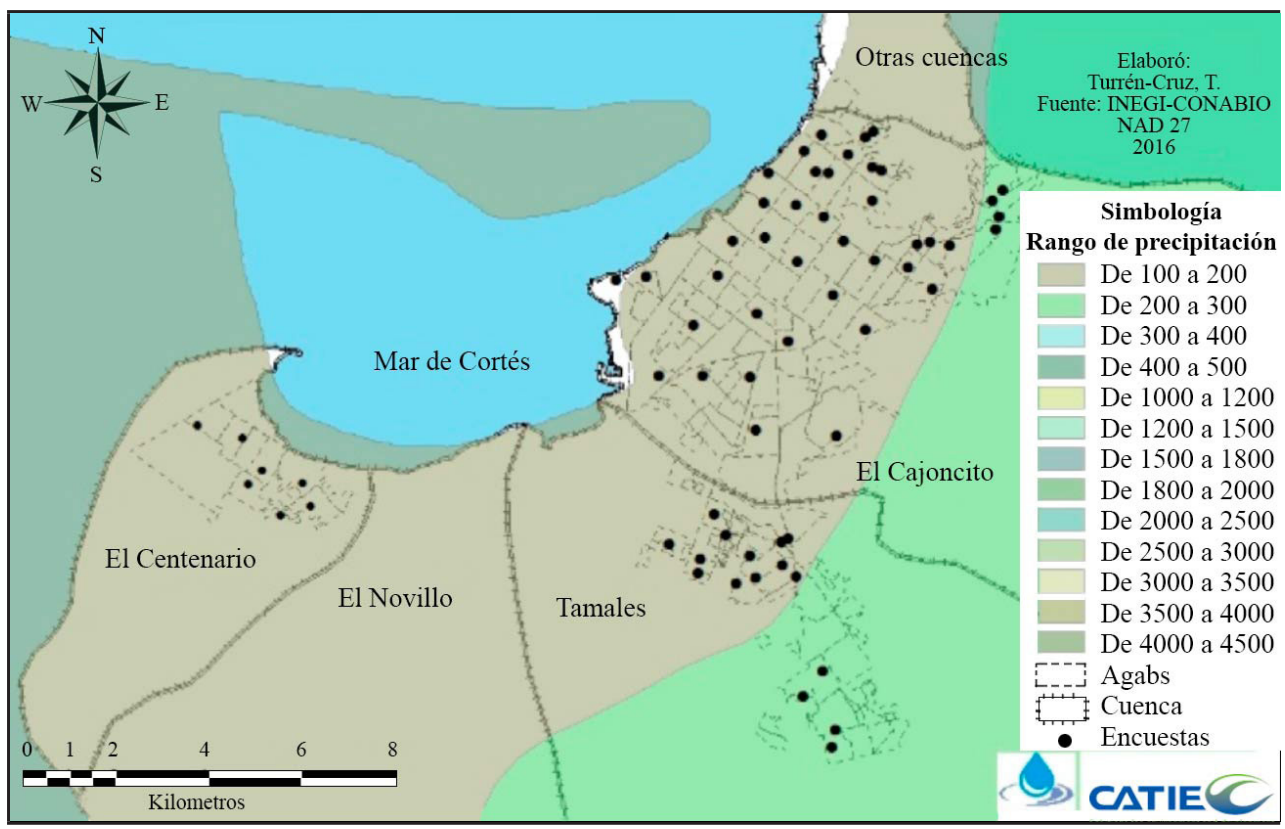

MAPA 12

Rango de precipitación en La Paz, BCS

Fuente: elaboración propia. 


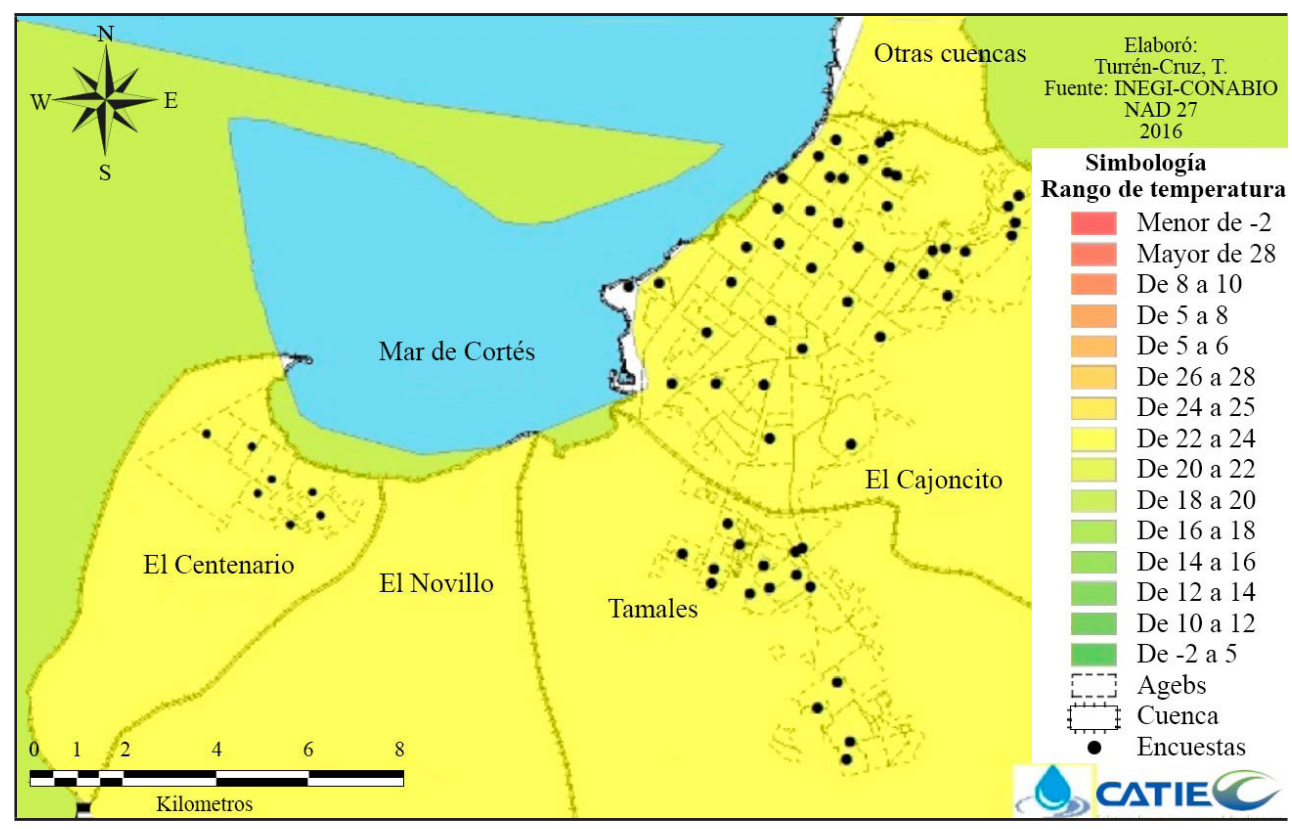

MAPA 13

Rango de temperatura en La Paz, BCS

Fuente: elaboración propia.

En general, se observa una alta vulnerabilidad global hacia las zonas periféricas más alejadas del centro de la ciudad; por ende, son las zonas más pobres, con menos acceso a diferentes servicios y con más deficiencias en el servicio y disponibilidad de agua potable. De acuerdo con Soares et al. (2014), los grupos marginados son los más susceptibles a sufrir daños en caso de amenazas, además la falta de sustentabilidad ambiental, el subdesarrollo y la pobreza son causas inevitables de los desastres. Entonces, con el fin de reducirlos hay que invertir en un sistema de desarrollo con más variables a monitorear que las meramente económicas, incluyendo las climáticas, culturales, sociales, entre otras (Lavell, 2000).

Diversos estudios realizados en la zona reflejan el problema del agua por la sobreexplotación, la baja precipitación y la intrusión salina. Se han buscado algunas soluciones como el tratamiento de aguas residuales y la instalación de medidores. Más allá de la percepción social analizada en este trabajo, la vulnerabilidad en La Paz está también dada por las fallas del gobierno federal, estatal y municipal en cuanto a la falta de cobro de concesiones a sus usuarios, falta de aplicación en la ley a los usuarios (mal manejo y desperdicio), necesidad de disminuir el desarrollo de asentamientos sin fuentes de agua suficientes, insuficiente conservación de zonas de recarga, mala administración y manejo del recurso debido a fugas, la falta de medición para hacer el cobro real del consumo y la eliminación de tarifas fijas o promediadas (Cruz Falcón y Troyo Diéguez, 2010a).

\section{Medidas y estrategias de respuesta para mejorar la capacidad adaptativa ante los EFECTOS DEL CAMBIO CLIMÁTICO}

La sociedad y los ecosistemas son usuarios clave de los recursos hídricos, puesto que trabajan como indicadores de su conducta, fortaleza y flexibilidad; representan también la capacidad de un sistema hídrico de enfrentar tensiones como la escasez del agua, degradación de la calidad, cambio climático o extracción excesiva. Esto define que las estrategias y medidas de respuesta varíen por sector de uso. La gestión de los recursos hídricos está ligada a la estimación de la disponibilidad de agua y también a la capacidad de adaptación de la sociedad y los ecosistemas. Desafortunadamente, se enfoca más en la disponibilidad de recursos hídricos que en cómo la sociedad y el ecosistema enfrentan la escasez de agua (Pandey et al., 2011). La adaptación se refiere a las capacidades de la 
sociedad y los ecosistemas para manejar sus recursos hídricos y heredarlos a las generaciones futuras (Pandey et al., 2011), es decir, adaptarse a las situaciones de escasez, sequía y exceso de agua (inundaciones).

Algunas investigaciones realizadas en la zona de La Paz recomiendan moderar el desarrollo comercial y turístico, mejorar la eficiencia en el tratamiento de aguas residuales, reparar la red de distribución de agua potable, proveer recarga artificial al acuífero, control de la extracción, planeación en el desarrollo de la ciudad, proteger zonas de recarga e instalar desaladoras (como último recurso), instalar medidores en toda la zona para calcular el consumo real y eliminar tarifas fijas, diseñar estrategias de captación de lluvia en los techos de casas y edificios, crear un instituto del agua que permita capacitar recursos humanos y diseñar un plan de manejo del agua (Cruz Falcón y Troyo Diéguez, 2010b; Cruz Falcón et al., 2009). Se sugiere además realizar capacitaciones con cierta periodicidad, con temas como el reúso del agua, qué hacer en caso de huracanes, cómo afrontar los efectos de la escasez de agua y sequía, crear un canal de flujo de información para conocer las necesidades de los habitantes y diseñar planes de acción, mejorar la infraestructura de distribución de agua, dar seguimiento y monitorear programas de gobierno, generar facilidades de acceso a crédito y, como medidas más rápidas, se puede crear un programa frecuente de entrega de pipas, así como la diversificación de actividades productivas; por ejemplo, como medida de adaptación en algunas zonas áridas se ha implementado la siembra de especies tolerantes a altas concentraciones de sal encontradas en el suelo derivado de sistemas de riego. Por ejemplo, se han usado especies como la cebada, el sorgo y el mijo (Flora y Thiboumery, 2005).

Incluir las aspiraciones, motivaciones y racionalidad de las comunidades en el proceso de estimación y disminución de la vulnerabilidad es un componente importante en la mejora de la conciencia de la problemática y en el aumento de capacidades para enfrentar, reducir, prevenir, mitigar y adaptarse a los efectos del cambio climático (Lampis, 2010), ya que no se puede hablar de adaptación de los pobladores ni de las comunidades si no conocemos el nivel de vulnerabilidad, que es el que nos permite plantear estrategias de adaptación acordes con la realidad y de implementación viable a nivel local (Ríos et al., 2011).

Gran parte de la efectividad de las acciones encaminadas a la prevención y recuperación depende de la respuesta social para que los impactos lleguen o no a ser fatales y además la toma de decisiones para la reducción de riesgos debe partir del grado de vulnerabilidad de un sistema, y no precisamente del daño que haya causado un evento, como suele asumirse, y se hace evidente en las políticas, acciones y presupuesto institucional aplicado que favorecen más la administración del desastre que la prevención (Ávila Flores y González Gaudiano, 2014).

Por tanto, para reducir la vulnerabilidad y minimizar impactos ante problemas de diversa índole se debe lograr que las comunidades sean más fuertes frente a estos eventos, ya que no se pueden controlar los efectos del clima mediante programas de prevención y monitoreo de los niveles de vulnerabilidad y adaptación al cambio climático.

Para combatir la vulnerabilidad habría que enfocarse en crear una integración sociedad-ecosistema-adaptación, donde corresponde tomar en cuenta que la capacidad adaptativa de una comunidad puede ser mejorada por el incremento en los flujos de información y conocimientos sobre elementos clave para la toma de decisiones: estado actual de sus capitales, redes, interacción y negociaciones entre instituciones a diferentes niveles, disponibilidad de recursos e igualdad (Engle y Lemos, 2010).

Conocer los niveles actuales de adaptación de La Paz y sus recursos hídricos provee una base para poder mejorarlos y, de acuerdo con este análisis, los habitantes no están preparados para enfrentar estas situaciones por la falta de infraestructura que permita manejar los grandes volúmenes de agua en temporada de huracanes y quizá con esto mitigar el efecto de la escasez y sequía en temporada de secas. Por sus condiciones geográficas, no se esperan mejoras en las condiciones climáticas; por lo tanto, la estimación de vulnerabilidad por capitales permite determinar los tipos de intervención política necesarias dentro de la ciudad para trabajar su capacidad adaptativa y recursos a fin de desarrollar nuevas estrategias de vida.

El uso del conocimiento generado y adoptado por la comunidad puede dar soporte y flexibilidad al aprendizaje social, lo cual se transformará mejorando y agilizando la toma de decisiones al momento del manejo y gestión de 
sus recursos. La generación de conocimiento en La Paz puede apoyarse del buen nivel de comunicación que hay a nivel institucional (capital político y cultural), además de aprovechar las facilidades tecnológicas, solamente falta crear programas o actividades que permitan esta comunicación para subsanar la relación comunidadgobierno y crear lazos de confianza y apoyo mutuo. Las instituciones y los mecanismos de gobernanza pueden facilitar o inhibir el éxito de las respuestas adaptativas y es necesario investigar qué mecanismos institucionales son los necesarios para construir capacidad adaptativa y cómo se relacionan e influencian unos a otros (Engle y Lemos, 2010). Esta investigación puede usarse como herramienta de análisis para obtener estos elementos y con seguridad se podrán fortalecer los capitales impactando positivamente la capacidad adaptativa para La Paz y para cualquier comunidad.

\section{Conclusiones}

El análisis de la vulnerabilidad ante los principales eventos climáticos desarrollados para La Paz, Baja California Sur, nos permite formular las siguientes conclusiones:

a) La metodología propuesta permite analizar una de las dimensiones de la vulnerabilidad al observar la percepción de la comunidad, así como plasmarla espacialmente para ayudar en la toma de decisiones o en el estudio de problemáticas definidas, además de identificar otros problemas potenciales de solucionar. El análisis a nivel local brinda mejores resultados que a escalas mayores donde se tiende a generalizar y resulta en pérdida de datos.

b) La representación de la vulnerabilidad a través de mapas es útil para exponer a la comunidad el estado actual de sus capitales, conocer cómo se comportan en diferentes zonas de la ciudad y además permitir el análisis de su nivel de capacidad adaptativa y resiliencia ante eventos climáticos. Esto puede proveerles un nivel de entendimiento base para mejorar en conjunto la gestión de sus recursos hídricos.

c) Desde la perspectiva de la zona en la que se ubica la ciudad de La Paz, no ha sido desarrollado un marco metodológico para medir la vulnerabilidad y la afectación de ésta a la capacidad adaptativa de la comunidad y sus recursos hídricos. Este estudio es un primer paso en esta dirección, así como la base para el desarrollo de estrategias de adaptación y llegar a un desarrollo sostenible.

d) Por su ubicación geográfica La Paz es vulnerable; esta vulnerabilidad la causa la escasez de agua y sequía, y además se suma la gestión hídrica ineficiente de las autoridades.

e) Todas las zonas requieren atención por parte de las autoridades. Las zonas periféricas de la ciudad serían las prioritarias, ya que presentan pérdida de vegetación, calidad del suelo y fauna. No cuentan con suficiente agua para satisfacer sus necesidades y la pérdida de trabajo afecta negativamente sus ingresos económicos.

f) El capital financiero se percibe como uno de los más vulnerables por afectaciones colaterales derivadas de los otros capitales; es decir, los demás capitales pueden estar afectando el capital financiero de una u otra forma, ya que las personas buscan siempre satisfacer primero sus necesidades en cuanto a vivienda y alimentación (recurso agua) invirtiendo en ellas sus recursos económicos.

g) No hay diferencias entre el mapa de vulnerabilidad global e integral debido a que desde un punto de vista climático existe homogeneidad en precipitación (de 100 a $300 \mathrm{~mm}$ ) y temperatura (de 22 a $24^{\circ} \mathrm{C}$ ) en la zona de estudio, y también porque la ciudad es pequeña y espacialmente similar.

b) La principal diferencia respecto a la vulnerabilidad la crea la percepción que la comunidad tiene ante eventos climáticos, por lo cual es indispensable trabajar con la comunidad en procesos participativos de planificación a través de grupos focales diferenciados por género, puesto que tanto hombres como mujeres pueden percibir de manera distinta la vulnerabilidad del recurso hídrico por su rol en sus 
medios de vida. Lo anterior para impactar además positivamente en la relación comunidad-gobierno, ya que está muy lastimada, no hay confianza y se demanda trabajo en conjunto.

i) El trabajo en conjunto permitirá involucrar a la comunidad en un proceso de análisis, diagnóstico, diseño, aplicación, evaluación y monitoreo de estrategias para actuar ente eventos climáticos provocando que se genere conocimiento que derive en la apropiación de las estrategias diseñadas.

j) Construir la comunicación entre la comunidad y las autoridades tomadoras de decisiones, y al mismo tiempo la creación de lazos de confianza es fundamental para enfrentar cualquier evento climático en La Paz.

k) Esta metodología es flexible y puede adaptarse a cualquier lugar haciendo un análisis previo para la selección de indicadores.

l) Es importante hacer notar que cada unidad de estudio es única y tendrá diferencias en sus capitales, por lo que la selección de indicadores depende del propósito del estudio, escala, tipo de zona/sistema bajo estudio, las preferencias del investigador y la disponibilidad de información.

m) Para el abordaje de la vulnerabilidad contextual y futura, se podrían incorporar escenarios de cambio climático en lugar del índice de Martonne como variable y analizar cómo a futuro podría cambiar la vulnerabilidad del territorio. También se podría realizar un análisis a futuro incorporando la modelación de escenarios de toma de decisiones, con modelos tipo WEAP, donde "incorporamos el futuro" con los escenarios de cambio climático y ensayamos los cambios que suponen las decisiones de manejo de la cuenca en función de ese escenario futuro.

n) Dentro del enfoque de resiliencia, se debería fortalecer este diagnóstico incluyendo umbrales y límites de cambio para la resiliencia que busquen la interacción instituciones-sociedad. Al final la resiliencia implica una acción política, institucional y social.

\section{Recomendaciones}

a) Realizar el análisis de incidencia climática por capital y no de manera global para posteriormente analizar las relaciones entre capitales en un mapa integrador final.

b) Usar esta información como herramienta base para la toma de decisiones, planeación y diseño de políticas públicas.

c) Realizar el análisis con perspectiva de género, esto es, desagregando la información que recoge cada indicador por género, ya que es probable que las percepciones de vulnerabilidad por capital serán diferentes si separamos los puntos de vista de hombres y mujeres. De esta manera, se contribuirá al diseño de políticas públicas desagregadas que aseguren la equidad de género.

d) Incluir el sector hotelero, así como toda la infraestructura relacionada con el sector turismo, dentro de los indicadores que evalúan el capital físico y también el sector salud, que tomó en cuenta la presencia de clínicas y cantidad de afiliados que evalúan el capital humano.

e) Mantener la homogeneidad en el número de indicadores por índice.

f) Considerar el número mínimo de indicadores, pero suficientes para representar el objeto de estudio. Si el índice pretende capturar mucha información puede incrementar la complejidad y crear problemas con el análisis e interpretación de los datos.

g) Para complementar el análisis de percepción social, se recomienda usar variables estadísticas de censos como la densidad poblacional y la población con servicios básicos como drenaje, entre otras, para optimizar el cálculo de los índices no sin antes homogenizar los datos.

b) La metodología propuesta no está sujeta a estudiar sólo eventos climáticos. Por el contrario, puede también aplicarse en análisis de gobernanza y análisis de prosperidad de agua.

i) Promover la aplicación de esta propuesta para validar los índices y resultados. 


\section{Agradecimientos}

Gracias al proyecto WaterClima-LAC, Gestión de zonas costeras (DCI-ENV/2014/350-470), por su apoyo financiero para este artículo, a los asesores del Centro Agronómico Tropical de Investigación y Enseñanza (CATIE) y al Consejo Nacional de Ciencia y Tecnología (CONACYT-COCYTECH) de México por el financiamiento de los estudios de maestría que derivan en esta investigación; por último, pero no menos importante, a todas las personas que de alguna forma tomaron unos minutos de su tiempo para ayudar a cumplir con los objetivos planteados.

\section{REFERENCIAS}

Aguilar, M. (2007). Vulnerabilidad y adaptación al cambio climático de los pobladores rurales de la planicie costera central de El Salvador. San Salvador: PNUD.

Alderman, M., Cohen, H. y Madhavan, S. (1988). Dietary sodium intake and mortality: the National Health and Nutrition Examination Survey (NHANES I). The lancet, 351.

Álvarez, G. (2014). Vulnerabilidad social y estrategias de gestión del riesgo de desastres en la cuenca Grijalva. México: IMTA/CATIE.

Ávila Flores, B. y González Gaudiano, É. (2014). Percepción social de los eventos climáticos extremos: una revisión teórica enfocada en la reducción del riesgo. Trayectorias, 16(39), 36-58.

Baca, M., Laderach, P., Haggar, J., Schroth, G. y Ovalle, O. (2014). An integrated framework for assessing vulnerability to climate change and developing adaptation strategies for coffee growing families in Mesoamerica. PLOS ONE, 9(2). 10.1371/journal.pone.0088463

Balzarini, M. G., González, L., M, Tablada, N., Casanoves, F., Di Rienzo, J. A. y Robledo, C. W. (2008). Infostat. Manual del usuario. Córdoba: Editorial Brujas.

Bouroncle, C., Imbach, P., Rodríguez-Sánchez, B., Medellín, C., Martinez-Valle, A. y Laderach, P. (2016). Mapping climate change adaptive capacity and vulnerability of smallholder agricultural livelihoods in Central America: ranking and descriptive approaches to support adaptation strategies. Climate Change, 141(1), 123-137.

Bruce, B. y Fries, J. (2003). The Stanford health assessment questionnaire: A review of Its history, issues, progress, and documentation. The Journal of Rheumatology.

CONABIO (Comisión Nacional para el Conocimiento y Uso de la Biodiversidad). (2010). Portal de geoinformación.

Cruz Falcón, A. y Troyo Diéguez, E. (2010a). El agua, ¿un recurso sustentable para La Paz? Análisis periodísticos B.C.S, 10(114), 16-17.

Cruz Falcón, A. y Troyo Diéguez, E. (2010b). Propuesta para solucionar el problema de agua en la ciudad de La Paz, B.C.S. Análisis periodísticos B.C.S, 119, 30-31.

Cruz Falcón, A., Troyo Diéguez, E. y Salinas González, F. (2009). Panorama de la problemática del agua en la ciudad de La Paz, B.C.S. Alternativa de Baja California, 71, 53-55.

Di Rienzo, J., Casanoves, F., Balzarini, M., Gonzalez, L., Tablada, M. y Robledo, C. (2016). InfoStat versión 2016. Argentina: Universidad Nacional de Córdoba.

Emery, M. y Flora, C. (2006). Spiraling-Up: mapping community transformation with comunity Capitals framework. Journal of Community Development Society, 37(1), 19-35.

Engle, N. L. y Lemos, M. C. (2010). Unpacking governance: Building adaptive capacity to climate change of river basins in Brazil. Global Environmental Change, 20(1), 4-13. 10.1016/j.gloenvcha.2009.07.001 
Escolero, O. y Torres-Onofre, S. (2007). Análisis de la intrusión de agua de mar en el acuífero de La Paz (México). Boletín Geológico y Minero, 118, 637-648.

ESRI. (2014). Box-Cox, arcsine, and log transformations. USA: ESRI.

Flora, C., Flora L, J. y Gasteyer P. S. (2016). Rural communities: legacy and change (5th edition). USA: Westview Press.

Flora, C. y Harris Gillespie, A. (2009). Making healthy choices to reduce childhood obesity: Community capitals and food and fitness. Community development, 40(2), 114-122.

Flora, C. y Thiboumery, A. (2005). Community capitals: poverty reduction and rural development in dry areas. Annals of arid zone, 3, 4(45), 239-253.

Flores Mora, C. B. M. (2014). Sequía histórica: cuatro países, 40 días sin lluvia, 2 millones con hambre en Centroamérica. Banco Mundial. Disponible en http://www.bancomundial.org/es/news/feature/2014/09/10/sequias-centroamerica

Fonseca, R. y Ramírez, A. (2014). Diagnóstico de las fuentes de abastecimiento de agua en La Paz, Baja California sur. XXIII Congreso Nacional de Hidráulica, 23, 8.

Gutiérrez-Montes, I., Emery, M. y Fernandez-Baca, E. (2009). The sustainable livelihoods approach and the community capitals framework: The importance of system-level approaches to community change efforts. Community development, 40(2), 106-113. 10.1080/15575330903011785

Gutierréz-Montes, I., Soares, D., Mareva, T., Galileo, G., Pinto, G., Ramirez, F.,... López, R. (2014). Análisis de la suceptibilidad de los recursos comunitarios ante eventos climáticos extremos en Sitalá, Chiapas: retos y propuestas conceptuales desde un enfoque de equidad social. Reflexiones y expresiones de la vulnerabilidad social en el sureste de México, 143-185.

Imbach, A. (2012). Estrategias de vida. Analizando las conexiones entre la satisfacción de las necesidades humanas fundamentales y los recursos de las comunidades rurales. Costa Rica: GeoLatina Ediciones.

INEGI (Instituto Nacional de Estadística y Geografía). (2010a). Cartas topográgficas: Baja California Sur. INEGI.

INEGI (Instituto Nacional de Estadística y Geografía). (2010b). Principales resultados por localidad. México: INEGI.

INEGI (Instituto Nacional de Estadística y Geografía). (2011). Panorama sociodemográfico de Baja California Sur/Instituto Nacional de Estadística y Geografía.

Lampis, A. (2010). Pobreza y riesgo medio ambiental: un problema de vulnerabilidad y desarrollo. Working paper. Centro Interdisciplinario de Estudios sobre Desarrollo CIDER.

Lampis, A. (2013). Vulnerabilidad y adaptación al cambio climático: debates acerca del concepto de vulnerabilidad y su medición. Cuadernos de Geografía, 22(2), 17-33.

Lavell, A. (2000). Desastres y desarrollo: hacia un entendimiento de las formas de construcción social de un desastre: el caso del huracán Mitch en Centroamérica. Ciudades en riesgo: degradación ambiental, riesgos urbanos y desastres. Universidad de Texas: BID/CIDHS.

Marateb Reza, J., Mansourian, M., Adibi, P. y Farina, D. (2014). Manipulating measurement scales in medical statistical analysis and data mining: A review of methodologies. Journal of Research in Medical Sciences, 19, 47-56.

Mercado Mancera, G., Troyo Diéguez, E., Aguirre Gómez, A., Murillo Amador, B., Beltrán Morales, L. y García Hernández, J. (2010). Calibración y aplicación del índice de aridez de de Martonne para el análisis del déficit hídrico como estimador de la aridez y desertificación en zonas áridas. Universidad y Ciencia, 26(1), 51-64.

Merrey, D. J., Drechsel, P., de Vries, F. W. T. P. y Sally, H. (2005). Integrating “livelihoods” into integrated water resources management: taking the integration paradigm to its logical next step for developing countries. Regional Environmental Change, 5(4), 197-204. 10.1007/s10113-004-0088-5. 
Murray, R. y Larry, J. (2005). Estadistica (cuarta edición). México.

ONU (Organización de las Naciones Unidas). (2014). Decenio Internacional para la Acción "El agua fuente de vida" 2005-2015. Disponible en http://www.un.org/spanish/waterforlifedecade/scarcity.shtml

Pandey, V. P., Babel, M. S., Shrestha, S. y Kazama, F. (2011). A framework to assess adaptive capacity of the water resources system in Nepalese river basins. Ecological Indicators, 11(2), 480-488. 10.1016/j. ecolind.2010.07.003

IPCC (Grupo Intergubernamental de Expertos del Cambio Climático) (2001). Cambio climático 2001: impactos, adaptación y vulnerabilidad. Tercer Informe de Evaluación del Grupo Intergubernamental de Expertos sobre el Cambio Climático.

Ríos, S., Louman, B. y Jiménez, M. (2011). Vulnerabilidad al cambio climático en comunidad indígenas cabécares de Costa Rica. Recursos naturales y ambiente, 63, 21-29.

Salas, M. y Jiménez, M. (2013). Inundaciones. México: CENAPRED.

SEGOB (Secretaría de Gobernación) (2013). Plan Nacional de Desarrollo. México.

Sibelet, N., Mutel, M., Arragon, P. y Luye, M. (2013). Los métodos de investigación cualitativa aplicada al manejo de los recursos naturales. Disponible en http://entretiens.iamm.fr/.

Soares, D. y Gutierréz-Montes, I. (2011). Vulnerabilidad social, institucionalidad y percepciones sobre el cambio climático: un acercamiento al municipio de San Felipe, Costa de Yucatán. CIENCIA ergo-sum, 18( 3), 249-263.

Soares, D., Millán, G. y Gutierréz-Montes, I. (2014). Reflexiones y expresiones de la vulnerabilidad social en el sureste de México. México: Instituto Mexicano del Agua.

Sseguya, H., Mazur, R. y Masinde, D. (2009). Harnessing community capitals for livelihood enhancement: experiences from a livelihood program in rural uganda. Community development, 40(2), 123-153.

Troyo Diéguez, E., Mercado Mancera, G., Cruz Falcón, A., Nieto Garibay, A., Valdez Cepeda, R., García Hernández, J. y Murillo Amador, B. (2015). Análisis de la sequía y desertificación mediante índices de aridez y estimación de la brecha hídrica en Baja California Sur, noroeste de México. Investigaciones Geográficas, 85. 10.14350/rig.32404

Wilches-Chaux, G. (1993). La vulnerabilidad global. Red de Estudios Sociales en Prevención de Desastres en América latina. s.l. CO.

\section{Notas}

[1] Esta fórmula se aplica para cada índice (siete capitales).

[2] Importancia baja: 1, importancia media: 2, importancia alta: 3 (las respuestas de los actores clave oscilaron siempre entre 2 y 3 , por lo cual el valor (1) de importancia baja no se observa en la tabla).

[3] Un enfoque para manejar variables ordinales es la introducción de una variable binaria ficticia al fusionar "siempre", "a veces" como "si" y "raramente", "nunca" como "no". De este modo, se puede usar una medida de distancia binaria adecuada (Marateb Reza et al., 2014).

[4] En este tipo de variable se considera un tipo de escala de calificación de cuatro ítems (por ejemplo: siempre, a menudo, a veces, nunca) que se ve ampliamente en los cuestionarios de nutrición gastrointestinal (Alderman et al., 1988) y la salud pública (Bruce y Fries, 2003).

[5] Disponible en http://www.worldclimate.com/

[6] a) Básicas: alimentación, salud, resguardo, reproducción y seguridad. b) De la persona: afecto, conocimiento, identidad, autoestima y responsabilidad.c) De entorno: ambiente saludable y libertad. d) De acción: trabajo creativo y productivo, recreación, participación y comunicación (Imbach, 2012).

\section{BY-NC-ND}

